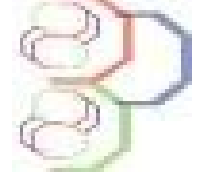

Journal of Applied Biosciences 133: 13592 - 13617

ISSN 1997-5902

\title{
Mushroom species richness, distribution and substrate specificity in the Kilum-ljim forest reserve of Cameroon
}

\author{
Ache Neh Teke1, Tonjock Rosemary Kinge ${ }^{2 *}$, Eneke Esoeyang Tambe Bechem ${ }^{1}$, Lawrence Monah \\ Ndam ${ }^{1,3}$ and Afui Mathias Mih ${ }^{1}$ \\ 1 Department of Botany and Plant Physiology, Faculty of Science, University of Buea, P.O. Box 63, South West \\ Region, Cameroon \\ 2 Department of Biological Sciences, Faculty of Science, The University of Bamenda, P.O. Box 39, Bambili, North \\ West Region, Cameroon \\ ${ }^{3}$ Tokyo University of Agriculture and Technology, International Environmental and Agricultural Sciences, Fuchu \\ Campus, Saiwai-cho, Fuchu, Tokyo 183-8509, Japan \\ Corresponding author: rosemary32us@yahoo.com
}

Original submitted in on $7^{\text {th }}$ November 2018. Published online at www.m.elewa.org/journals/ on $31^{\text {st }}$ January 2019 https://dx.doi.org/10.4314/jab.v133i1.11

\begin{abstract}
Objectives: Mushroom species richness, distribution and substrate specificity are essential considerations for conservation and management of forest ecosystems. In this study in the Kilum-ljim mountain forest, mushroom species richness, distribution and substrate specificity was evaluated.

Methodology and results: Fixed size plot method, opportunistic and downed wood sampling methods according to Mueller et al. (2004) was used for mushroom survey in 2015 in five sites in the Kilum-ljim forest. The number of species, diversity in each site as well as substrate and morphotypes were recorded and calculated. A total of 393 macrofungi samples were collected. Highest collection (135) was recorded in September while January registered the least (22). Plantlife Sanctuary Oku recorded the highest number of macrofungal (109) while the least (44) was recorded in Anyajua community forest. Macrofungi were found on five varying substrates and ten different morphological forms of macrofungal were recorded.

Conclusions and application of findings: Mushrooms species richness differed in the five sites surveyed in the Kilum-ljim forest. Podoscypha petalodes had the highest number of species across all the five sites followed by Coprinus fissolanatus, Polyporus dictopus, Favolachia calocera and Xylaria sp. The Agaricaceae and Polyporaceae were the most abundant family. Majority of the mushroom species were found on dead wood substrate and few on dung and standing tree substrates. The result on species richness, distribution and substrate specificity of mushrooms widens the knowledge on mycodiversity and substrate relationship which is an important factor for conservation and utilization as well as for the sustainable forest ecosystem management. Also, the result projects the importance of geographic location of substrates on the distribution of mushrooms which is of immense value for conservation. There is variation in the substrate preferred by mushrooms and likely this factor influences the extent of occurrence and nutritional content.
\end{abstract}

Keywords: Mushrooms, Species richness, Substrate, Distribution, Kilum-ljim 


\section{INTRODUCTION}

Macrofungi are one of the richest and diverse groups of organisms on earth and constitute a significant part of terrestrial ecosystem, forming a large share of species richness and key players of ecosystem processing (Seen-Irlet et al., 2007). Macrofungi play essential roles in maintaining forest ecosystems and biodiversity (Hawksworth, 1991; Molina et al., 2008). Macrofungi substrate refers to any surface on which the macrofungi attach and grow whereas the macrofungi include fungi distinguished by having fruiting structures visible to the naked eye commonly referred to as mushrooms (Lodge et al., 2004). Most terrestrial macrofungi are decomposers, but some species form mycorrhiza and a few are parasitic. Fungi fruiting on woody substrata are usually either saprobes or plant pathogens (Mueller et al., 2007). Macrofungi play significant roles as an integral part of the forest ecosystem. Forest trees form a relationship with some species of macro-fungus (called mycorrhizae) that helps tree roots absorb water and minerals from the soil. Mycorrhiza constitutes the most efficient nutrient uptake facilitators particularly in nutrient deficient soils of tropical regions where it enhances mineral uptake by connecting the plant's roots to a huge nutrient absorbing mycelial network which solubilize minerals such as phosphate, making it available for absorption (Onguene and Kuyper, 2001; Eneke, 2011). Forestry managers and conservationists have realized that dead decaying wood which is mostly facilitated by fungi, forms an important source of biodiversity and an integral part of the recycling of carbon and other nutrients (Gates, 2009). Macrofungi are important source of food for forest animals and they serve as homes for many soil insects and other small organisms that are also part of a healthy forest ecosystem. Moreover, macrofungi fruit bodies have enormous use for the general welfare in human life (Boa, 2004). They are highly useful in pharmaceutical industry and in mass production of cultivated fungi in the food industry (Lindequist et al., 2005), playing vital roles in biodegradation and biodeterioration (Tibuhwa, 2011). Macrofungi comprise different morphological forms. These include the rusts, smuts, jelly fungi, club fungi, coral and shelf (bracket) fungi, mushrooms, puffballs, stinkhorns and bird's-nest fungi. Morphologically they can be broadly divided into the following forms; bracket shaped, stiped and poroid, gilled, smooth andresupniate on woods, coralloid, hood like, dentoid or with cup shaped cap having or lacking stipe (Pacioni and Lincoff, 1981). Species diversity is one of the most obvious and characteristic feature of a community (Krebs, 2014). Diversity comprises two separate ideas-species richness and evenness. Species richness is the oldest and simplest concept of species diversity. It measures the different kinds of organisms present in a community or region. McIntosh (1967) coined the name species richness to describe this concept. Species evenness compares the similarity of the population size of each of the species present. Lloyd and Ghelardi (1964) were the first to suggest the concept of evenness. In Cameroon, there are few community level studies of macrofungi such as the work of Egbe et al. (2013) in the Mount Cameroon Region, Kinge et al. (2017) who documented mushrooms in the Awing Forest Reserve. Also, Douanla-Meli (2007) studied the ecological diversity of mushrooms from the Mbalmayo forest reserves with emphasis on the taxonomy of non-gilled Hymenomycetes. 271 distinct species belonging to 110 genera in 58 families were recorded. Many new records and species new to science and important ethnomycological notes for people in and around the Mbalmayo forest reserves were documented. Roberts and Ryvarden (2006), reported over 70 poroid species was from the Korup rainforest, Cameroon. There are also single genus studies in Cameroon such as Kinge et al. (2012) who worked on the genus Ganoderma. Mossebo et al. (2009), and Mossebo et al. (2017) worked on the taxonomy genus Temitomyces from Cameroon. Despite the importance of macrofungi in natural and agroecosystems, there is scanty information on macrofungi community structure, substrate specificity and dynamics especially in the tropics 
(Hawksworth, 1991; Osemwegie et al., 2006; Muller et al., 2007). Fungal diversity and substrate specificity are usually overlooked during management of forest ecosystems, (Amaranthus, 1998; Tibuhwa, 2011); yet successful conservation of any ecosystem requires understanding of

\section{MATERIALS AND METHODS}

Study area: The Kilum Mountain Range and the ljim Ridge are situated in the Northwest Region of Cameroon commonly called the Bamenda Highlands. The Kilum-ljim forest (KIF) is located between Latitude $6^{\circ} 07^{\prime} \mathrm{N}$ and $6^{\circ} 17^{\prime} \mathrm{N}$ and Longitude $10^{\circ} 20^{\prime} \mathrm{E}$ and $10^{\circ} 35^{\prime} \mathrm{E}$ covering an area of about 20,000 ha. It is found on Mount Oku with Lake Oku lying in a crater in its centre. At 3,011 m, Mount Oku is the second highest mountain in West Africa, after Mount Cameroon (Fomété et al., 2001). The forest within this area borders three districts: Oku, Kom, and Nso. Part of the forest that borders Oku is called Kilum, and the rest that borders Kom and Nso is called ljim - hence, jointly the Kilum-ljim forest. The Kilum-ljim forest has been mushroom communities in terms of ecology and distribution. This study therefore aimed at determining the species richness, distribution and substrate specificity of mushrooms in the Kilumljim forest in the North West Region of Cameroon.

demarcated in to 18 community forests to enable better management of the forest resources by the communities surrounding the forest (Gardner et al., 2001). The climate of the Kilum Mountain is very humid with very high presence of fog and mist almost throughout the year (Fomété et al., 2001). The precipitation is unimodal (Asanga, 2001). The dry season begins from November to mid-March and the rainy season of 8 months long starts from mid-March to the month of October (Asanga, 2001). The total annual rainfall varies from $1800 \mathrm{~mm}$ to $3000 \mathrm{~mm}$ annually, with an average temperature that varies from $22^{\circ} \mathrm{C}$ at $1800 \mathrm{~m}$ altitude to $16^{\circ} \mathrm{C}$ in the higher altitude areas Figure 1.

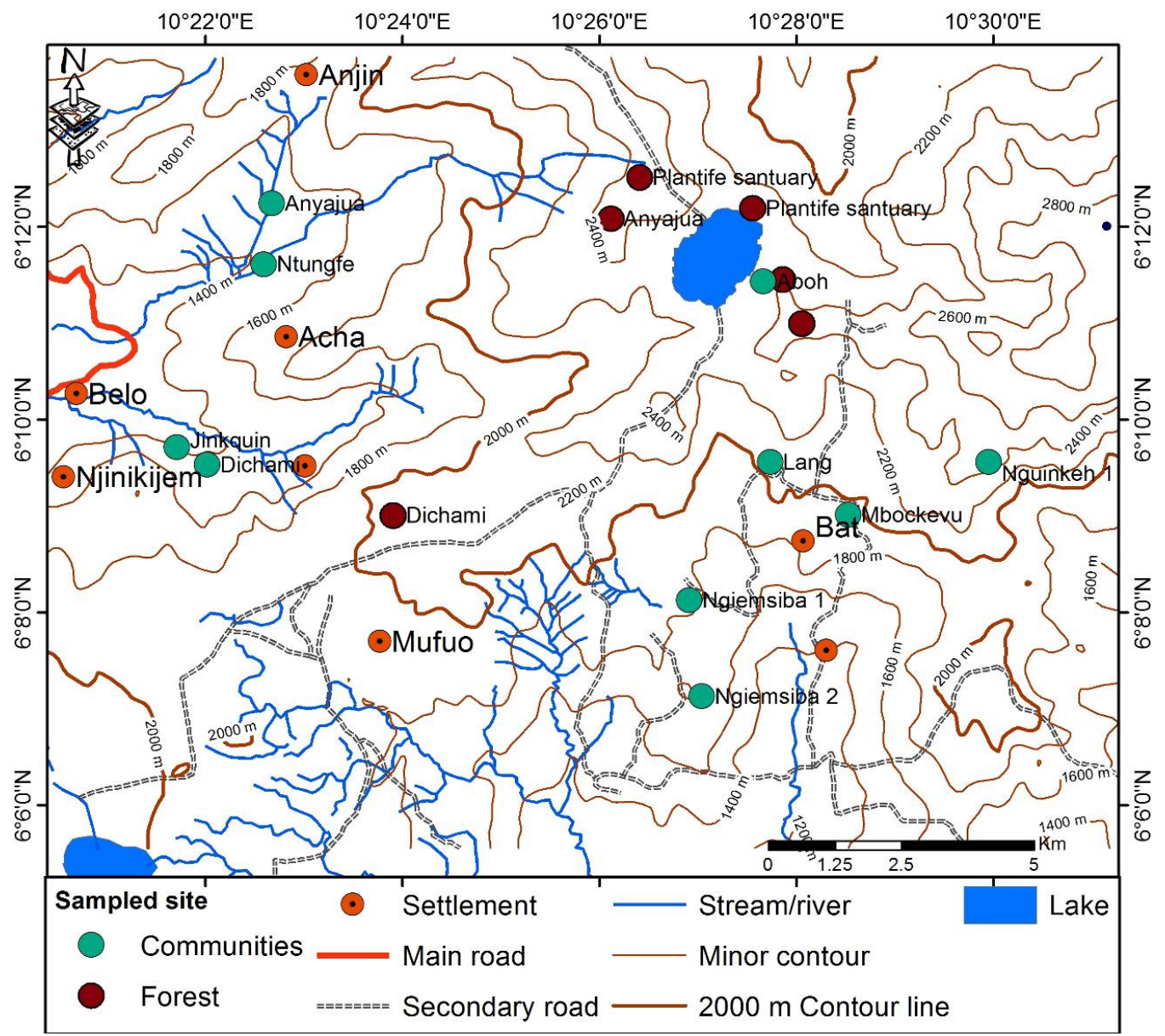




\section{Ache et al., J. Appl. Biosci. 2019 Mushroom species richness, distribution and substrate specificity in the Kilum-ljim forest reserve of Cameroon}

Figure 1: View of the Kilum-ljim mountain forest, Northwest Region Cameroon

The topography of the area is hilly and constitutes a chain of mountains. The highest of these mountains is mount Oku. The geological landscape found here are mainly of Basalts, trachytes, rhyolites, gneiss and granite origin. The soils here are humified ferralitic soils with a high organic matter content favored by the humid climate and cold. These soils are well drained and of good permeability (Fomété et al., 2001). The montane forest has a unique ecosystem that provides a favorable milieu for the habitation of many endemic plant and bird species (Asanga, 2001). The area is one of the most densely populated parts of Cameroon. It is estimated that close to 300000 people live within a day's walk of the forest (Forboseh and Maisels, 2000). The majority of the area enclosed by the Kilum-ljim boundary is at an altitude of over 2000 meters. The vegetation is at this altitude and above consists mainly of montane forest mixed with montane grassland and subalpine communities. Below this, most of the submontane forest has already disappeared due to clearance for agriculture.

Field survey and Sampling: Diversity study was carried out in five community forests of Kilum-ljim (Figure 2). Before entering into the Kilum-ljim forest, visitations were made to the fondoms and administrative authorities within the Kom and Oku districts to seek traditional and administrative permission to use the forest. Five community forests out of 18 were selected based on accessibility after a reconnaissance survey was carried out in the area.

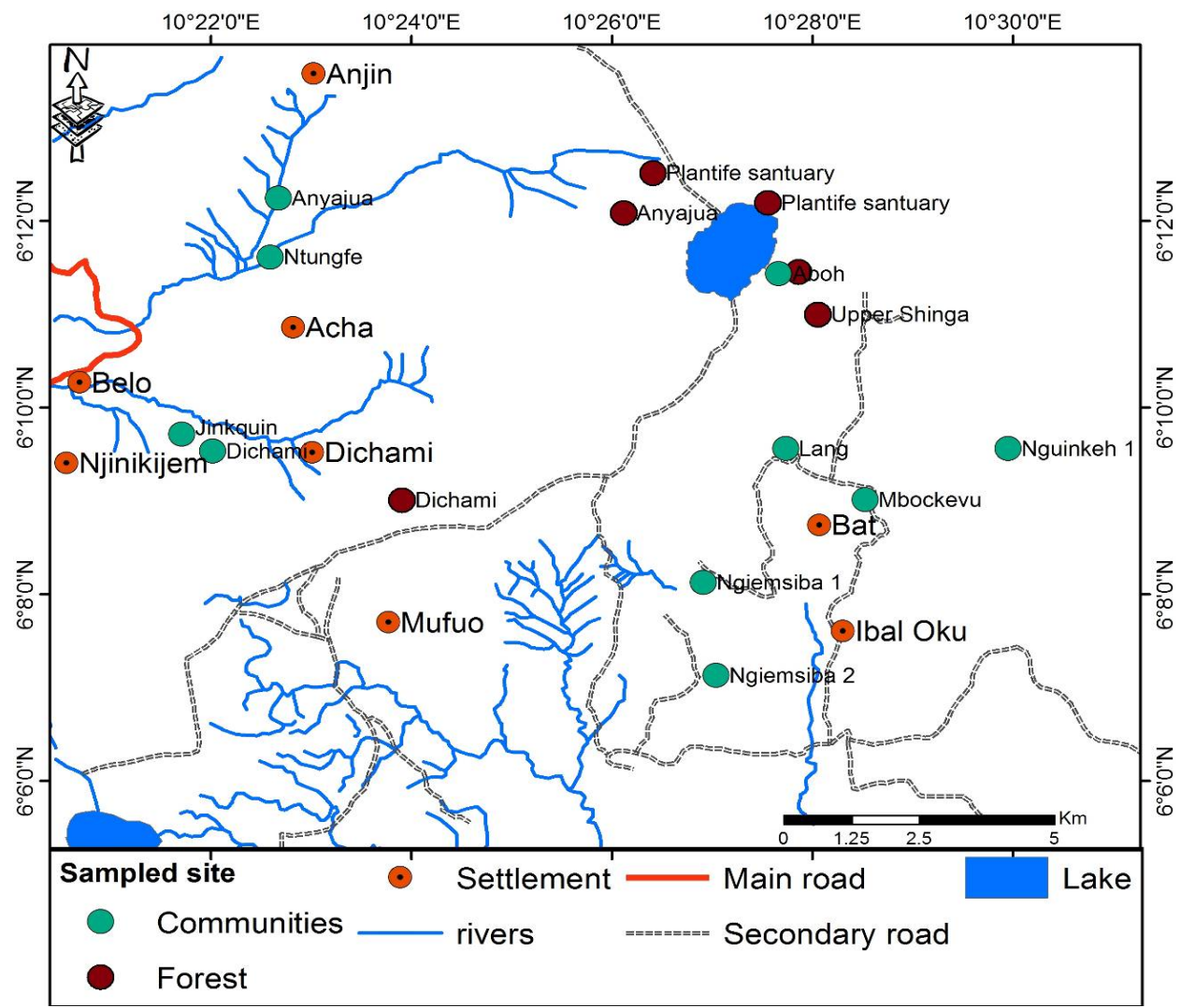

Figure 2: Macrofungal species richness and distribution sites in Kilum-ljim forest.

One reconnaissance survey and four field surveys were conducted in five community forests within the Kilumljim forest ridge. These included the Plantlife sanctuary (PLSB), Dichami community forest (DCF) and Anyajua community forest (ACF) in Belo subdivision, and Plantlife sanctuary (PLSO) and Upper shinga community forest (USCF) in Bui subdivision (Table 1). 
Table 1: Locational coordinates of macrofungi sampling sites in Kilum-ljim forest

\begin{tabular}{l|c|c}
\multicolumn{1}{c|}{ Sampling site } & Altitude (m) & Surface area (ha) \\
\hline Plantlife sanctuary Belo (PLSB) & 2421 & 1081 \\
Anyajua community forest (ACF) & 2497 & 1121 \\
Dichami community forest (DCF) & 2032 & 1034 \\
Plantlife sanctuary Oku (PLSO) & 2267 & 1081 \\
Upper-Shinga community forest (USCF) & 2455 & 1556 \\
\hline
\end{tabular}

Source: Gardner et al., 2001

The reconnaissance survey took place in November 2014 while the species richness survey trips were carried out in January, March, June, September and November 2015. These periods marked the beginning, middle and end of fructification season of different morphological types of macrofungi and each trip lasted seven days. Sampling methods comprised fixed size plot method, opportunistic sampling method and downed wood sampling method. These sampling methods according to Mueller et al. (2004), optimizes the number of macrofungal species recorded at any given site. For each sampling site, two plots of $50 \mathrm{X}$ $20 \mathrm{~m}$ were mapped out since all the studied sites were above 1000 ha. Each plot was separated by a distance of $200 \mathrm{~m}$. Each plot was $1000 \mathrm{~m}$ in length. A total of 10 plots were surveyed in all the five sampling sites. To capture maximum diversity, opportunistic sampling and downed wood sampling were carried out in and out of the plots. Coordinates of macrofungi sites were taken by the use of Garmin Etrex Venture Geographical Positioning System (GPS). For each macrofungus collected, the fungus was labelled and the growth substrate recorded. The morphological characters were assessed. These included cap shape, stipe length, size and color recorded. This information was used to place the fungus on different morphotypes according to Mueller et al. (2007). Photographs of labelled samples were recorded since some features can change during preservation. Macrofungal samples were dug off the soil or cut off with a knife where they were found on wood substrata. The samples were wrapped with their tags using aluminum foil and put in zip lock bags for drying. Drying at $45^{\circ} \mathrm{C}-55^{\circ} \mathrm{C}$ was done in a locally designed open air oven for up to 72 hours for thick samples. The number of samples collected in each site and sampling period were compared using the $x_{j}^{2}$ statistics.

Estimation of diversity and similarity: The number of species in each sampling site was recorded and the diversity and similarity indices between sites were calculated using Simpson diversity index and Jaccard's similarity index, respectively. Simpson's Diversity Index, 1-D, was estimated as follows:

$$
\begin{aligned}
& 1-D=1-\frac{\sum n(n-1)}{N(N-1)} \\
& n=\text { the number of individuals of each different } \\
& \text { species } N=\text { the total number of individuals of all the } \\
& \text { species }
\end{aligned}
$$

The Jaccard similarity coefficient was estimated as follows:

$J(A, B)=\frac{\mathrm{A} \cap \mathrm{B}}{\mathrm{A} \cup \mathrm{B}}=\mathrm{A} \cap \frac{\mathrm{B}}{(\mathrm{A})}+(\mathrm{B})-(\mathrm{A} \cup \mathrm{B})$

Where $A$ and $B$ are the number of species in each sample. 
Ache et al., J. Appl. Biosci. 2019 Mushroom species richness, distribution and substrate specificity in the Kilum-ljim forest reserve of Cameroon

\section{RESULT}

Distribution of Macrofungi in Different Sampling Sites in the Kilum-ljim Forest: Macrofungi were recorded at all the five sites studied in the KIF. They occurred in various shapes sizes and colors (Figure 3).
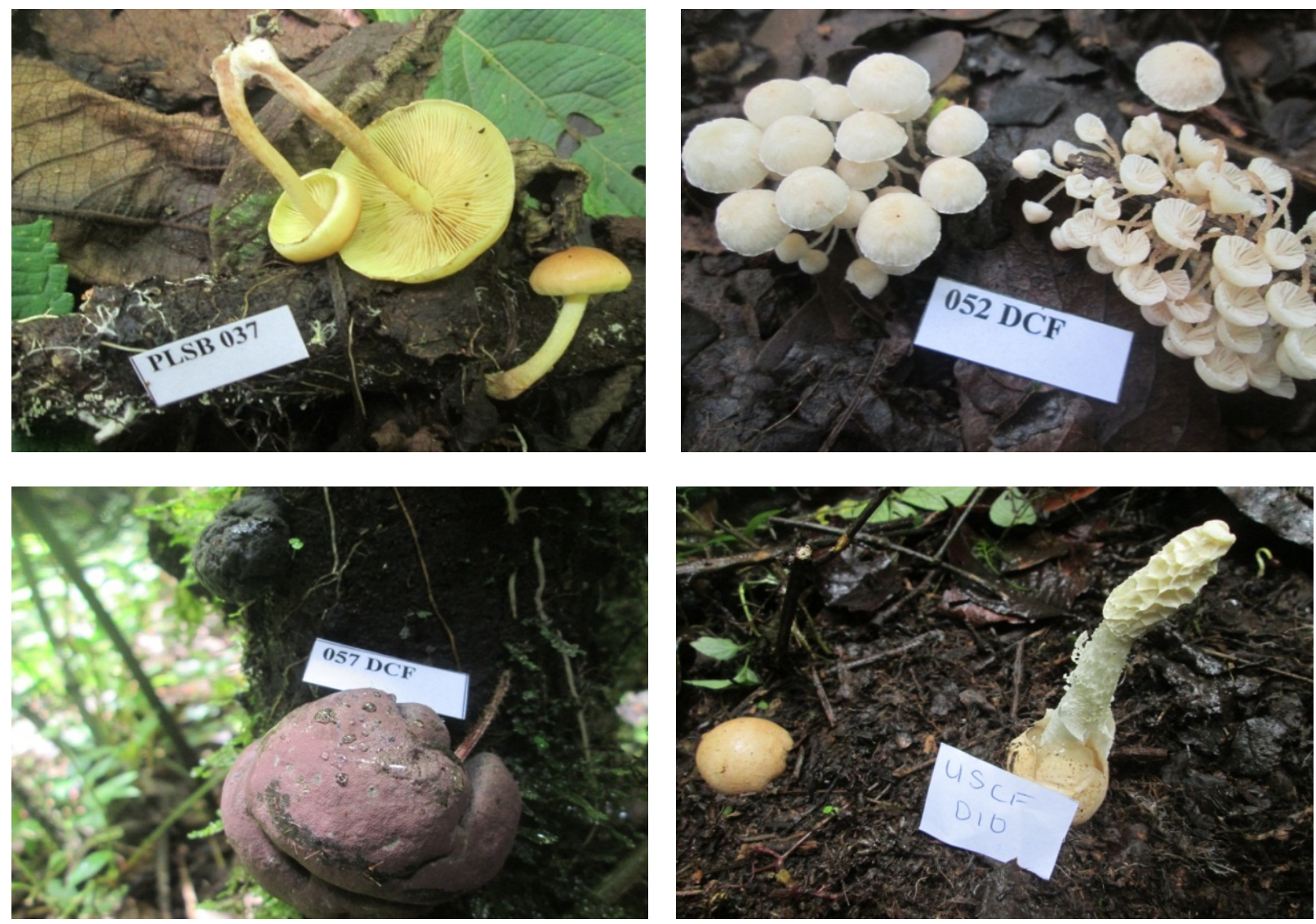

Figure 3: Tagged macrofungi samples of various shapes in their natural habitats

A total of 393 macrofungi samples were collected in the five forest sites studied (Table 2). Highest collection (135) was recorded in the month of September while January registered the least number of macrofungi (22). Plantlife Sanctuary Oku recorded the highest number of macrofungal samples (109) while the least number (44) was recorded in Anyajua community forest. Except for the collection of June 2015, there were no significant differences in the number of samples collected per site. Meanwhile there were significant differences in number of samples collected per sampling date in all the sites except in Anyajua community forest.

Table 2: Collection frequency of macrofungi samples in five sites of the KIF over different sampling periods

\begin{tabular}{|c|c|c|c|c|c|c|c|}
\hline \multirow{2}{*}{ Month/Year } & \multicolumn{5}{|c|}{ Location } & \multirow{2}{*}{ Total } & \multirow{2}{*}{$X^{2}$} \\
\hline & PLSB & ACF & DCF & PLSO & USCF & & \\
\hline January 2015 & 06 & 03 & 05 & 06 & 02 & 22 & 0.929 \\
\hline March 2015 & 17 & 07 & 14 & 13 & 07 & 58 & 5.077 \\
\hline June 2015 & 27 & 10 & 24 & 31 & 11 & 103 & $18.341^{*}$ \\
\hline September 2015 & 21 & 15 & 32 & 37 & 30 & 135 & 10.207 \\
\hline November 2015 & 19 & 09 & 14 & 22 & 11 & 75 & 6.218 \\
\hline Total & 90 & 44 & 89 & 109 & 61 & 393 & \\
\hline$x^{2}$ & $19.422^{*}$ & 5.167 & $24.522^{*}$ & $33.598^{*}$ & $37.152^{*}$ & & \\
\hline
\end{tabular}




\section{Ache et al., J. Appl. Biosci. 2019 Mushroom species richness, distribution and substrate specificity in the Kilum-ljim forest reserve of Cameroon}

*= Significant difference $(p<0.05)$

$\mathrm{PLSB}=$ Plantlife sanctuary Belo, $\mathrm{ACF}=$ Anyajua community forest, $\mathrm{DCF}=$ Dichami community forest, $\mathrm{PLSO}=$ Plantlife sanctuary Oku, USCF= Upper-shinga community forest

Macrofungi occurred in soil, deadwood, leaf litter, cow dung and on some standing stems. A total of 218 samples were collected from dead wood while cow

dung registered the least number $(03)$ of macrofungi. (Table 3).

Table 3: Occurrence of Macrofungi on different substrates in the different sampling sites

\begin{tabular}{|c|c|c|c|c|c|c|c|}
\hline \multirow{2}{*}{ Substrate } & \multicolumn{5}{|c|}{ Location } & \multirow{2}{*}{ Total } & \multirow{2}{*}{$x^{2}$} \\
\hline & PLSB & ACF & DCF & PLSO & USCF & & \\
\hline Deadwood & 49 & 28 & 47 & 57 & 37 & 218 & $11.633^{*}$ \\
\hline Leaf Litter & 24 & 14 & 28 & 22 & 13 & 101 & 8.356 \\
\hline Soil & 15 & 06 & 13 & 16 & 15 & 65 & 5.077 \\
\hline Cow Dung & 00 & 00 & 03 & 00 & 00 & 03 & 0.000 \\
\hline On standing stem & 02 & 00 & 01 & 02 & 01 & 06 & 0.667 \\
\hline Total & 90 & 48 & 92 & 97 & 66 & 393 & \\
\hline$x^{2}$ & $54.489^{*}$ & $15.500^{*}$ & $80.391^{*}$ & $67.660^{*}$ & $37.152^{*}$ & & \\
\hline
\end{tabular}

* = Significant difference $(p<0.05)$

$\mathrm{PLSB}=$ Plantlife sanctuary Belo, $\mathrm{ACF}=$ Anyajua community forest, $\mathrm{DCF}=$ Dichami community forest, $\mathrm{PLSO}=$ Plantlife sanctuary Oku, USCF= Upper-shinga community forest

Morphological diversity of macrofungi: Ten different morphological forms of macrofungi were encountered in the Kilum-ljim forest. These were the gilled fungi (60\%), Earthstar fungi, Polypores fungi, Crampball fungi, Coral fungi, Jelly fungi, Stinkhorns fungi, Bird's nest fungi, and Cup fungi which together constituted $40 \%$ of all the collections (Table 4).

Table 4: Morphoforms and site location of the different macrofungi occurring in the Kilum-ljim forest.

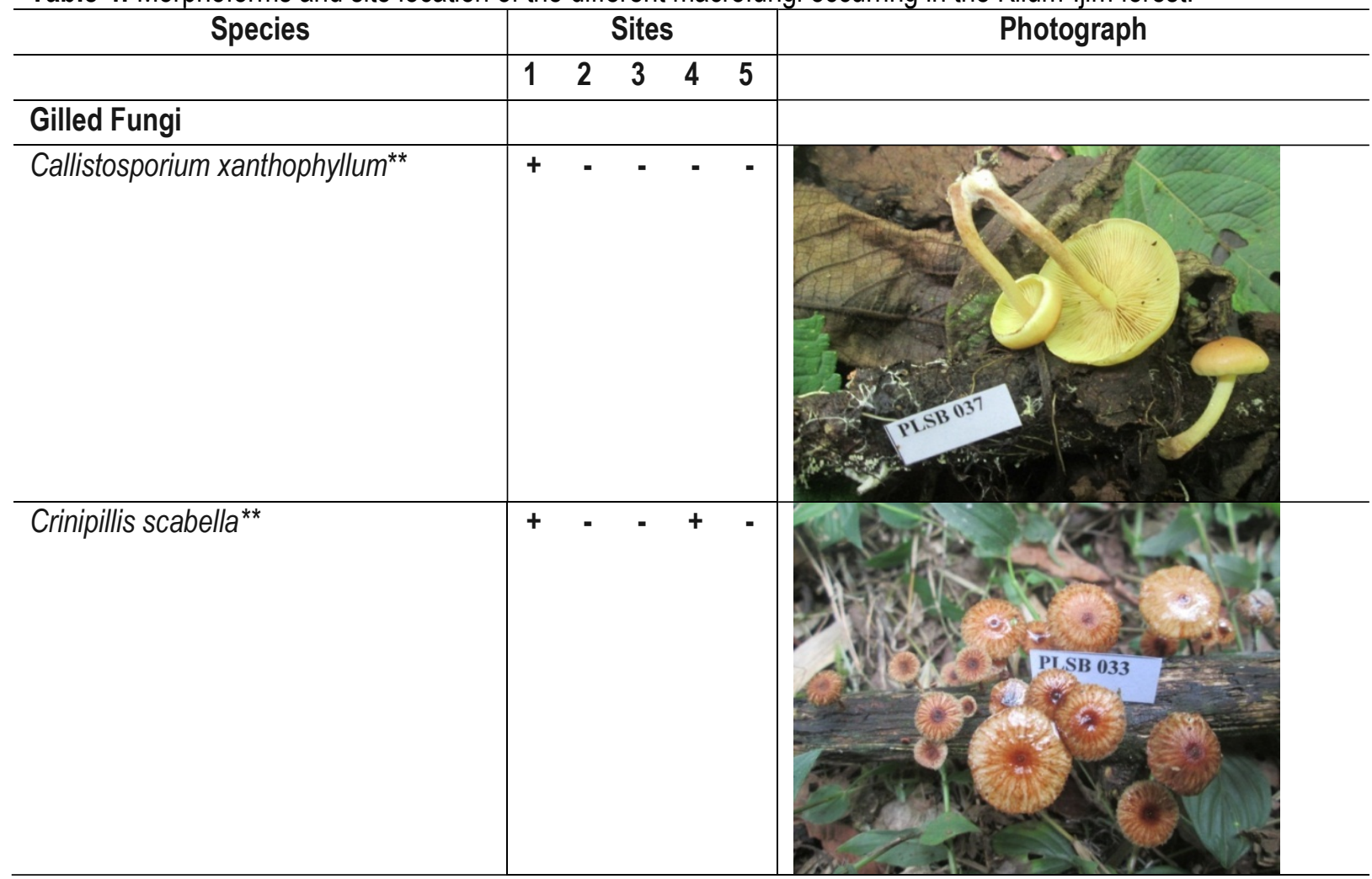


Ache et al., J. Appl. Biosci. 2019 Mushroom species richness, distribution and substrate specificity in the Kilum-ljim forest reserve of Cameroon

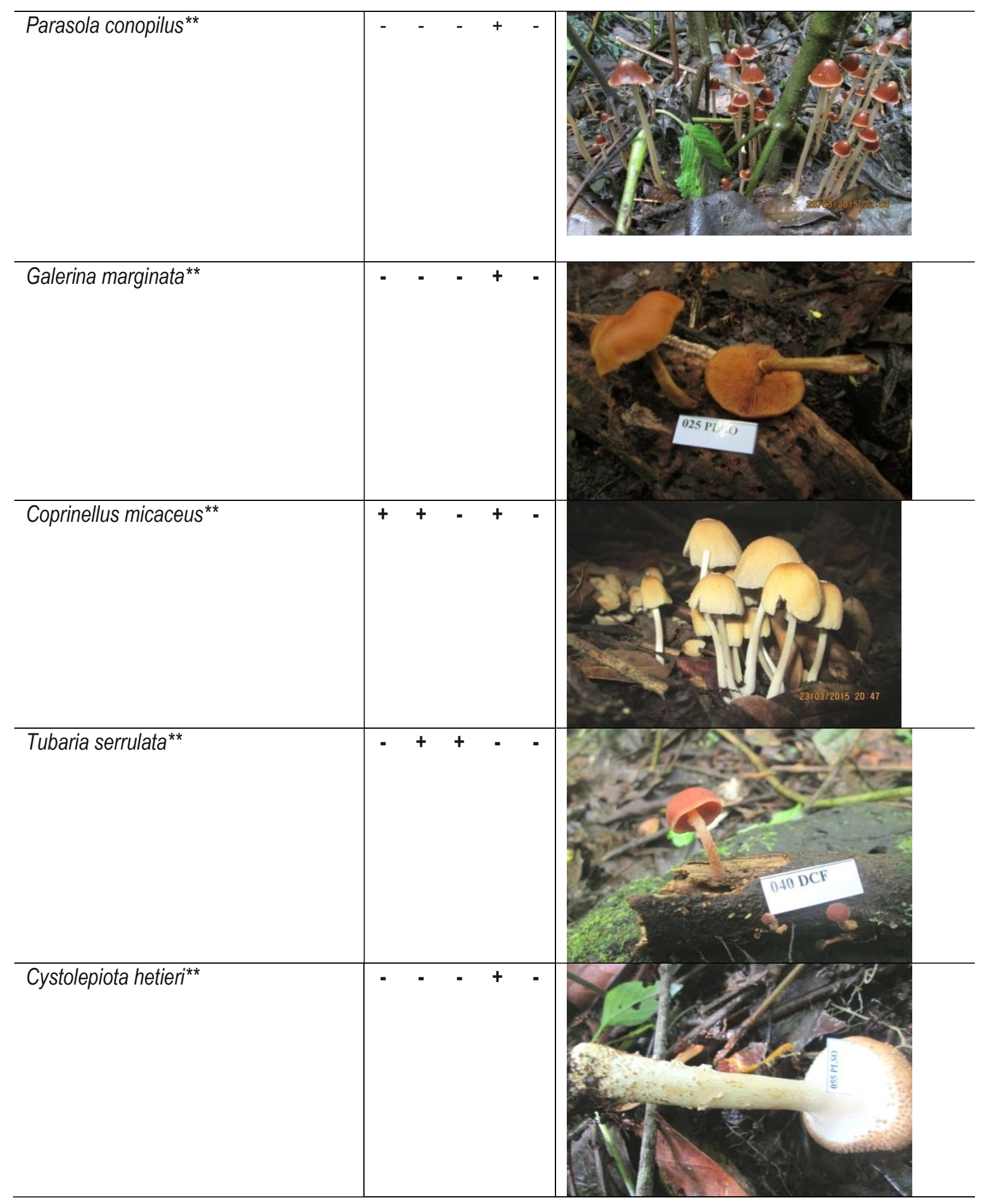


Ache et al., J. Appl. Biosci. 2019 Mushroom species richness, distribution and substrate specificity in the Kilum-ljim forest reserve of Cameroon

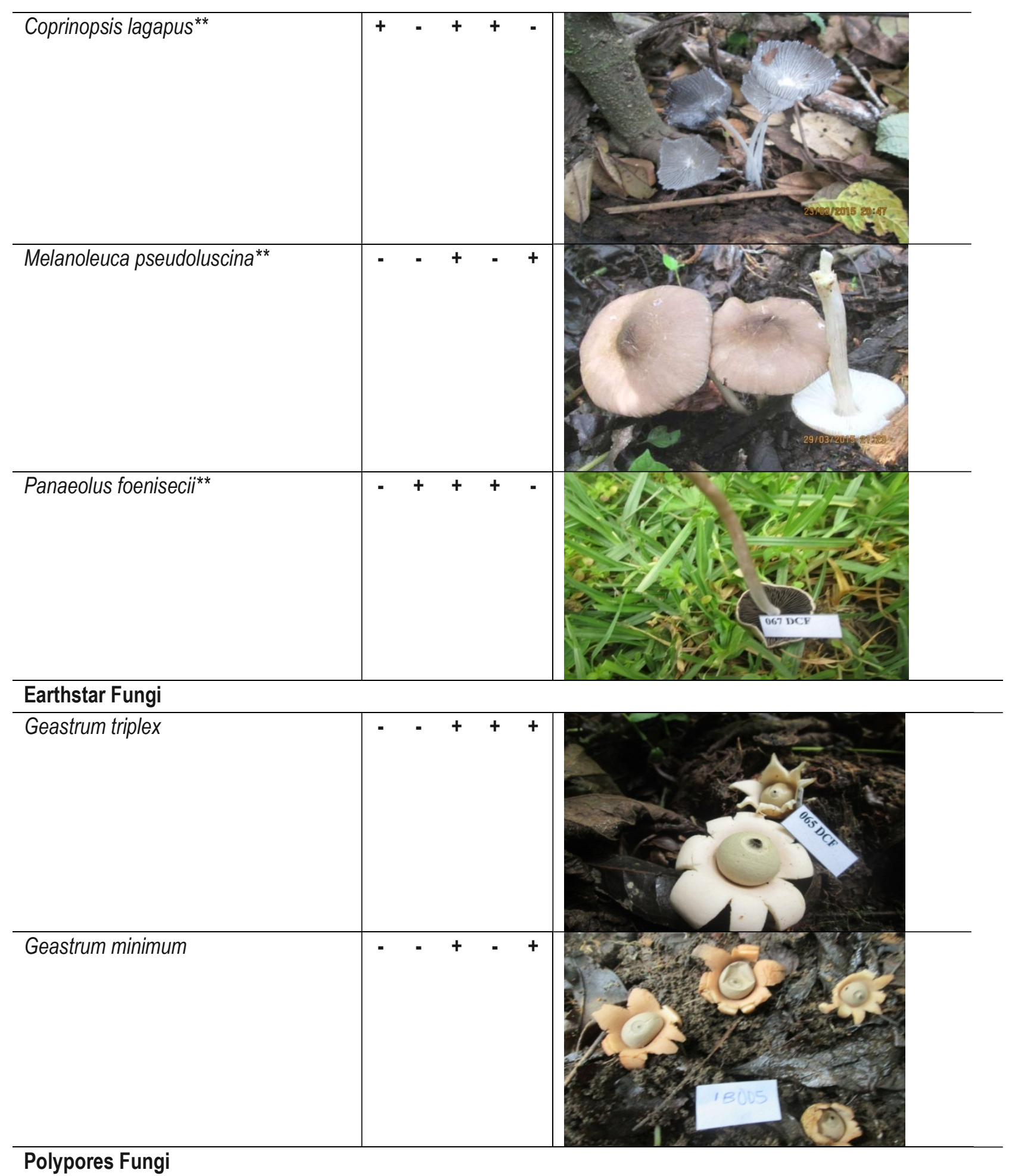


Ache et al., J. Appl. Biosci. 2019 Mushroom species richness, distribution and substrate specificity in the Kilum-ljim forest reserve of Cameroon

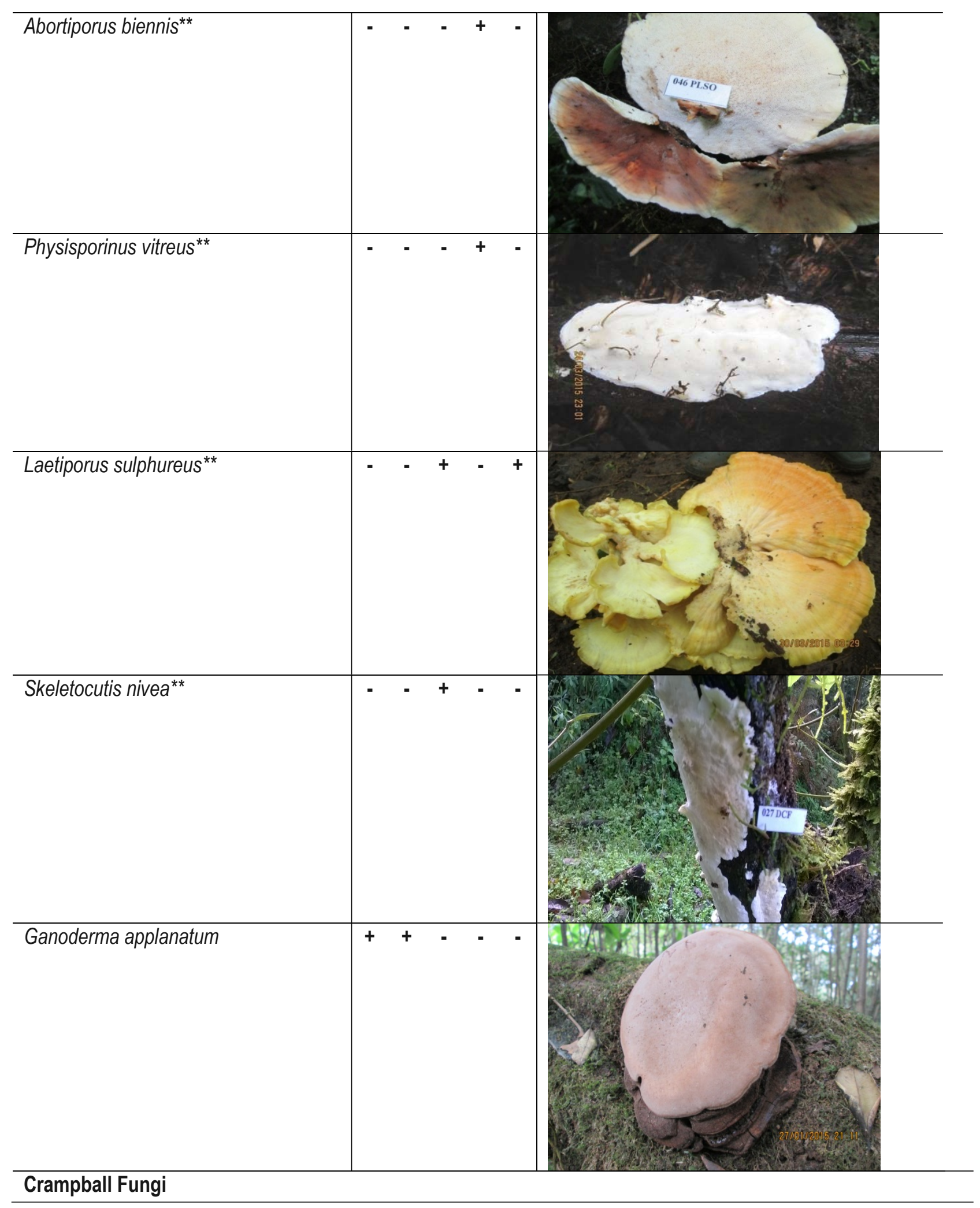


Ache et al., J. Appl. Biosci. 2019 Mushroom species richness, distribution and substrate specificity in the Kilum-ljim forest reserve of Cameroon

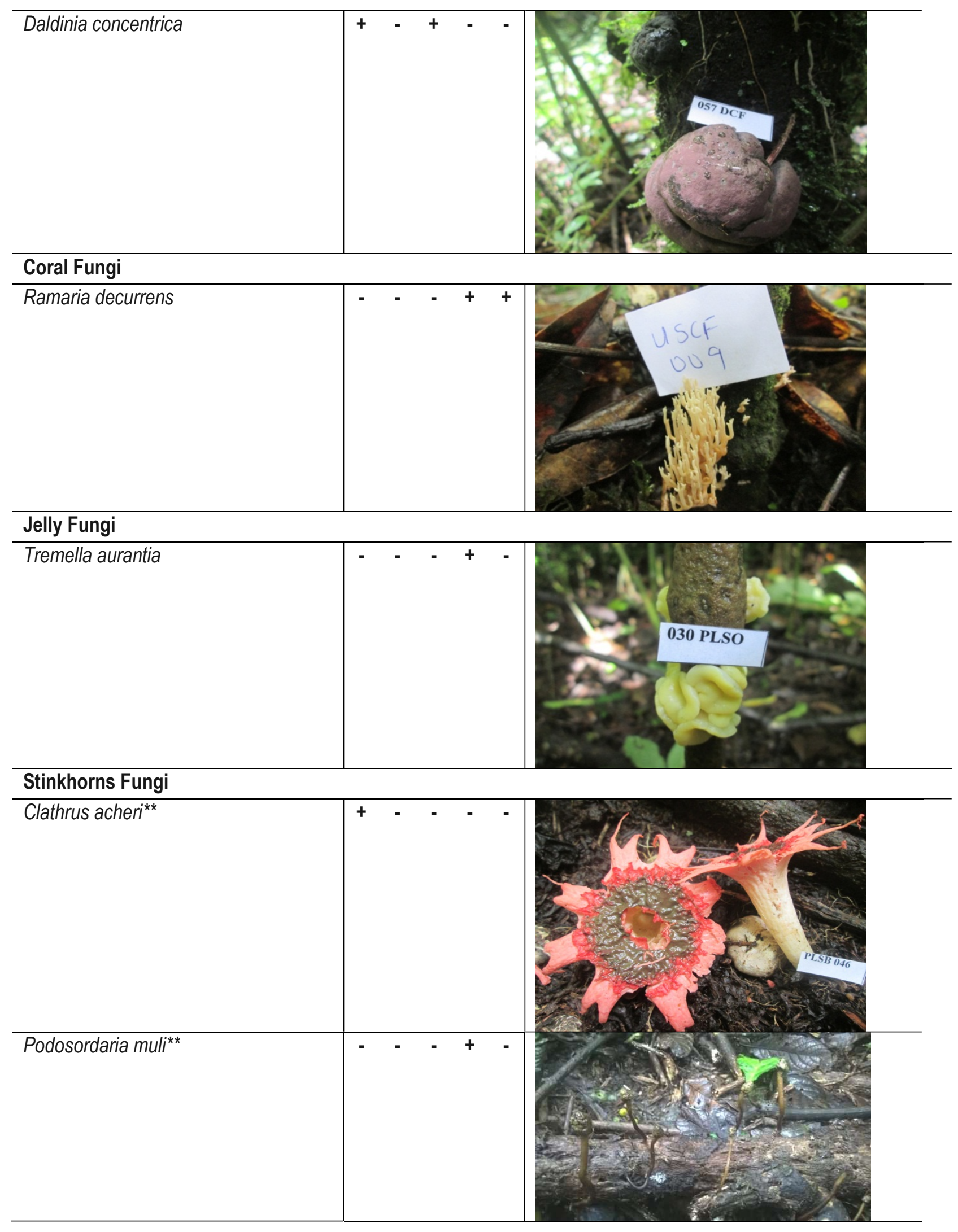



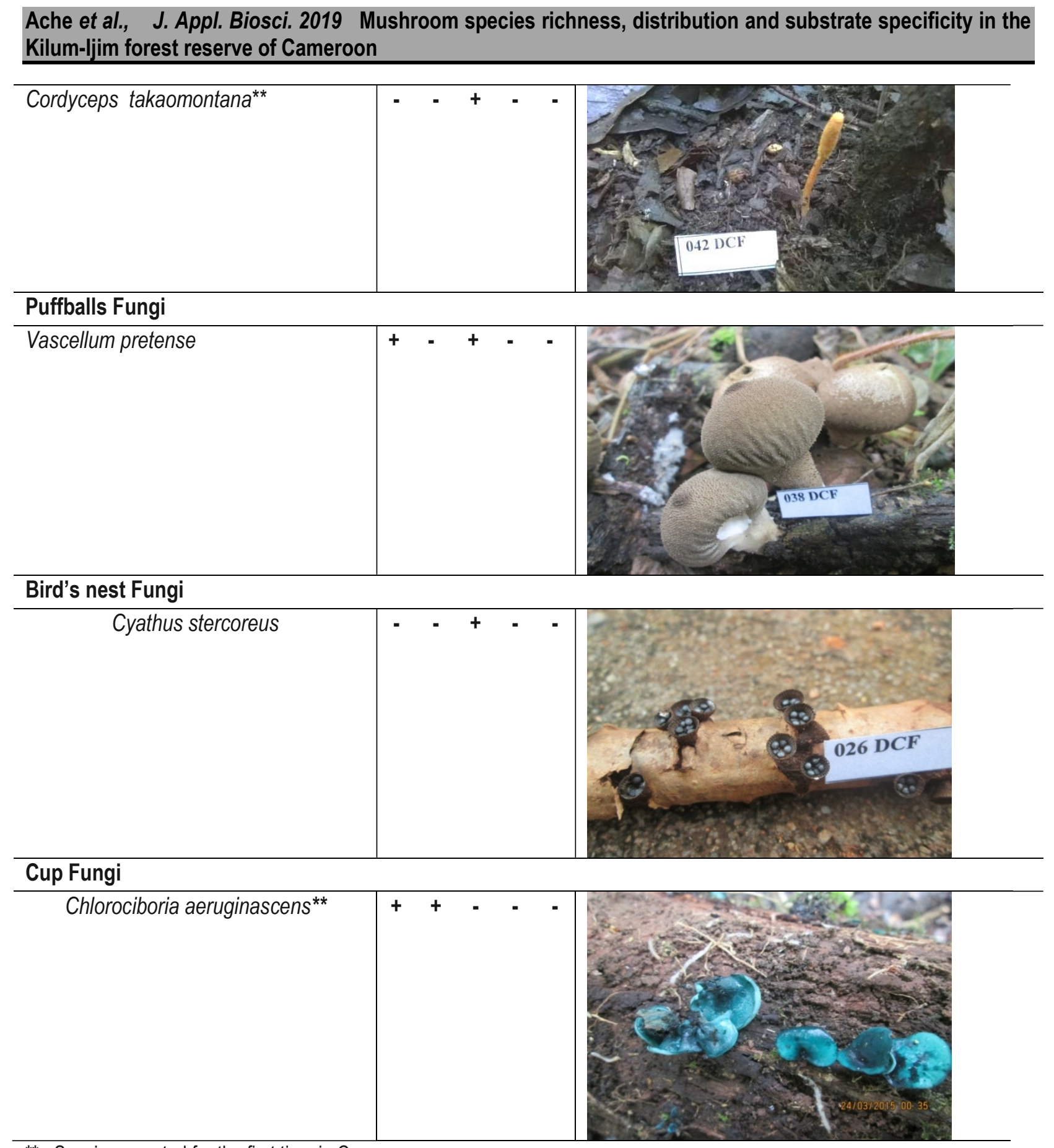

** $=$ Species reported for the first time in Cameroon

Site 1= Plantlife sanctuary Belo, Site 2= Anyajua community forest, Site 3= Dichami community forest, Site 4= Plantlife sanctuary Oku, Site $5=$ Upper-shinga community forest

Plantlife Sanctuary Oku (PLSO) had the highest number of mushroom species (86) and the site with the least number of species (35) was Anyajua Community Forest (ACF). Podoscypha petalodes had the highest number of species across all the five sites surveyed (11) followed by Coprinus fissolanatus (10), Polyporus dictopus (9) and Favolaschia calocera and Xylaria sp. with 8 species each Table 5 . 
Table 5: Frequency of occurrence of macrofungi collected from different sampling sites in the Kilum-ljim forest

\begin{tabular}{|c|c|c|c|c|c|c|c|c|}
\hline \multirow[b]{2}{*}{$S / N$} & \multirow[b]{2}{*}{ Species } & \multirow[b]{2}{*}{ Accession number } & \multicolumn{5}{|c|}{ Frequency } & \multirow[b]{2}{*}{ TOTAL } \\
\hline & & & PLSB & ACF & DCF & PLSO & USCF & \\
\hline & AGARICALES & & & & & & & \\
\hline 1 & $\begin{array}{c}\text { Agaricaceae } \\
\text { Agaricus litoralis (Wakef. \& } \\
\text { A. Pearson) Pilat. }\end{array}$ & JN204436 & 0 & 0 & 1 & 0 & 0 & 1 \\
\hline 2 & $\begin{array}{c}\text { Agaricus xanthodermus } \\
\text { Genev. (1876) }\end{array}$ & EU326208 & 0 & 0 & 1 & 0 & 0 & 1 \\
\hline 3 & $\begin{array}{c}\text { Cystolepiota hetieri (Boud.) } \\
\text { Singer }\end{array}$ & AY176459 & 0 & 0 & 0 & 2 & 0 & 2 \\
\hline 4 & $\begin{array}{c}\text { Lepiota sp PA620 (Pers.) } \\
\text { Gray (1821) }\end{array}$ & EF527355 & 1 & 0 & 0 & 0 & 1 & 2 \\
\hline 5 & $\begin{array}{l}\text { Leucoagaricus cupresseus } \\
\text { (Burl.) Boisselet \& Guinb. }\end{array}$ & GU139787 & 1 & 0 & 1 & 2 & 0 & 4 \\
\hline 6 & $\begin{array}{c}\text { Leucoagaricus flavovirens } \\
\text { J.F. Liang, Zhu L. Yang \& } \\
\text { J. Xu }\end{array}$ & EU416295 & 1 & 0 & 0 & 0 & 0 & 1 \\
\hline 7 & $\begin{array}{l}\text { Leucoagaricus gaillardii } \\
\text { Bon \& Boiffard } 1974\end{array}$ & GQ329042 & 0 & 0 & 1 & 0 & 0 & 1 \\
\hline 8 & $\begin{array}{l}\text { Leucoagaricus littoralis } \\
\text { (Menier) Bon \& Boiffard } \\
1970\end{array}$ & GQ329041 & 0 & 0 & 1 & 1 & 0 & 2 \\
\hline 9 & $\begin{array}{l}\text { Leucoagaricus rubrotinctus } \\
\text { (Peck) Singer (1948) }\end{array}$ & JN944081 & 0 & 0 & 0 & 0 & 1 & 1 \\
\hline 10 & $\begin{array}{l}\text { Leucoagaricus serenus } \\
\text { (Fr.) Bon \& Boiffard } 1974\end{array}$ & AY176420 & 0 & 1 & 0 & 1 & 0 & 2 \\
\hline 11 & $\begin{array}{l}\text { Leucoagaricus viriditinctus } \\
\text { (Berk. \& Broome) J.F. } \\
\text { Macrolepiota dolichaula }\end{array}$ & EU419375 & 0 & 0 & 0 & 1 & 0 & 1 \\
\hline 12 & $\begin{array}{l}\text { (Berk. \& Broome) Pegler \& } \\
\text { R.W. Rayner }\end{array}$ & JQ683120 & 1 & 1 & 2 & 0 & 1 & 5 \\
\hline 13 & $\begin{array}{l}\text { Vascellum pretense (Pers.) } \\
\text { Kreise }\end{array}$ & FJ481033 & 1 & 0 & 1 & 0 & 0 & 2 \\
\hline 14 & $\begin{array}{l}\text { Panaeolus foenisecii } \\
\text { (Pers.) R.Maire (1933). }\end{array}$ & JF908520 & 0 & 1 & 1 & 3 & 0 & 5 \\
\hline 15 & $\begin{array}{c}\text { Panaeolus sphinctrinus } \\
\text { (Fr.) Quél. } \\
\text { Crepidotaceae }\end{array}$ & JF908513 & 1 & 0 & 1 & 0 & 0 & 2 \\
\hline 16 & $\begin{array}{c}\text { Crepidotus epibryus (Fr.) } \\
\text { Quél. } 1888\end{array}$ & HM240524 & 1 & 1 & 2 & 2 & 1 & 7 \\
\hline 17 & $\begin{array}{c}\text { Crepidotus mollis } \\
\text { (Schaeff.) Staude } 1857 \\
\text { Entolomataceae }\end{array}$ & JF907959 & 0 & 0 & 0 & 1 & 0 & 1 \\
\hline 18 & $\begin{array}{l}\text { Entoloma araneosum } \\
\text { (Quél.) M.M. Moser. }\end{array}$ & EU784204 & 0 & 0 & 0 & 0 & 1 & 1 \\
\hline
\end{tabular}




\begin{tabular}{|c|c|c|c|c|c|c|c|c|}
\hline \multirow[b]{2}{*}{$S / N$} & \multirow[b]{2}{*}{ Species } & \multirow[b]{2}{*}{ Accession number } & \multicolumn{5}{|c|}{ Frequency } & \multirow[b]{2}{*}{ TOTAL } \\
\hline & & & PLSB & ACF & DCF & PLSO & USCF & \\
\hline 19 & $\begin{array}{c}\text { Hygrophoraceae } \\
\text { Camarophyllus pratensis } \\
\text { (Pers.) P. Kumm. }\end{array}$ & FJ596880 & 0 & 1 & 0 & 0 & 0 & 1 \\
\hline 20 & $\begin{array}{l}\text { Hygrocybe helobia } \\
\text { (Arnolds) Bon }\end{array}$ & JF908056 & 0 & 0 & 0 & 0 & 2 & 2 \\
\hline 21 & $\begin{array}{l}\text { Hygrocybe persistens } \\
\text { (Britzelmayr) Singer }\end{array}$ & FM208893 & 0 & 0 & 0 & 0 & 1 & 1 \\
\hline 22 & $\begin{array}{c}\text { Hymenogastraceae } \\
\text { Galerina badipes (Pers.) } \\
\text { Kühner }\end{array}$ & JF908012 & 1 & 0 & 0 & 1 & 0 & 2 \\
\hline 23 & Galerina hybrida Kühner. & AJ585445 & 0 & 0 & 1 & 0 & 0 & 1 \\
\hline 24 & $\begin{array}{c}\text { Galerina marginata } \\
\text { (Batsch) Kühner (1935) }\end{array}$ & AF501564 & 0 & 0 & 1 & 0 & 0 & 1 \\
\hline 25 & $\begin{array}{c}\text { Psilocybe cubensis (Earle) } \\
\text { Singer } \\
\text { Lyophyllaceae }\end{array}$ & HM035082 & 1 & 0 & 1 & 1 & 0 & 3 \\
\hline 26 & $\begin{array}{c}\text { Lyophyllum connatum } \\
\text { P.Karst. }\end{array}$ & JF908332 & 0 & 1 & 0 & 0 & 0 & 1 \\
\hline 27 & $\begin{array}{l}\text { Termitomyces microcarpus } \\
\text { (Berk. \& Broome) R.Heim }\end{array}$ & AF357023 & 0 & 0 & 0 & 0 & 3 & 3 \\
\hline 28 & $\begin{array}{l}\text { Termitomyces striatus } \\
\text { (Beeli) R.Heim }\end{array}$ & AF321367 & 0 & 0 & 0 & 4 & 0 & 4 \\
\hline 29 & $\begin{array}{c}\text { Termitomyces sp V1P } \\
\text { R.Heim }\end{array}$ & JF302830 & 0 & 0 & 0 & 0 & 1 & 1 \\
\hline 30 & $\begin{array}{c}\text { Termitomyces sp Group8 } \\
\text { R.Heim } \\
\text { Marasmiaceae }\end{array}$ & AB073529 & 0 & 0 & 0 & 1 & 0 & 1 \\
\hline 31 & $\begin{array}{c}\text { Clitocybula lacerata } \\
\text { (Scop.) Singer ex Métrod }\end{array}$ & FJ596916 & 1 & 1 & 0 & 2 & 0 & 4 \\
\hline 32 & $\begin{array}{c}\text { Clitocybula oculus (Peck) } \\
\text { Singer } 1962\end{array}$ & DQ192178 & 1 & 1 & 2 & 3 & 0 & 7 \\
\hline 33 & $\begin{array}{c}\text { Crinipellis scabella (Alb. \& } \\
\text { Schwein.) Murrill }\end{array}$ & JF907969 & 1 & 0 & 0 & 1 & 0 & 2 \\
\hline 34 & $\begin{array}{l}\text { Hydropus marginellus } \\
\text { (Pers.: Fr.) Singer } 1948 \\
\text { Marasmius }\end{array}$ & EU669314 & 0 & 0 & 0 & 0 & 1 & 1 \\
\hline 35 & $\begin{array}{c}\text { purpureostriatus Hongo } \\
1958\end{array}$ & FJ904978 & 0 & 1 & 2 & 0 & 0 & 3 \\
\hline 36 & $\begin{array}{l}\text { Marasmiellus ramealis } \\
\text { (Bull.) Singer }\end{array}$ & JF313670 & 2 & 0 & 3 & 1 & 0 & 6 \\
\hline 37 & $\begin{array}{c}\text { Marasmius rotula (Scop.) } \\
\text { Fr. } \\
\text { Mycenaceae }\end{array}$ & JN714927 & 2 & 0 & 0 & 4 & 0 & 6 \\
\hline 38 & $\begin{array}{c}\text { Favolaschia calocera R. } \\
\text { Heim }\end{array}$ & EU489640 & 1 & 0 & 4 & 0 & 3 & 8 \\
\hline 39 & $\begin{array}{c}\text { Mycena acicula (Schaeff.) } \\
\text { P.Kumm. (1871) }\end{array}$ & JF908384 & 1 & 1 & 0 & 4 & 1 & 7 \\
\hline
\end{tabular}


Ache et al., J. Appl. Biosci. 2019 Mushroom species richness, distribution and substrate specificity in the Kilum-ljim forest reserve of Cameroon

\begin{tabular}{|c|c|c|c|c|c|c|c|c|}
\hline \multirow[b]{2}{*}{$S / N$} & \multirow[b]{2}{*}{ Species } & \multirow[b]{2}{*}{ Accession number } & \multicolumn{5}{|c|}{ Frequency } & \multirow[b]{2}{*}{ TOTAL } \\
\hline & & & PLSB & ACF & DCF & PLSO & USCF & \\
\hline 40 & $\begin{array}{c}\text { Mycena laevigata (Lasch) } \\
\text { Gillet }\end{array}$ & JF908397 & 0 & 0 & 0 & 1 & 0 & 1 \\
\hline 41 & $\begin{array}{c}\text { Mycena pura (Pers.) P. } \\
\text { Kumm. }\end{array}$ & EU517506 & 0 & 1 & 2 & 0 & 0 & 3 \\
\hline 42 & $\begin{array}{c}\text { Panellus stipticus (Bull.) } \\
\text { P.Karst. (1879) } \\
\text { Nidulariaceae }\end{array}$ & FJ481038 & 0 & 0 & 1 & 0 & 0 & 1 \\
\hline 43 & $\begin{array}{c}\text { Cyathus stercoreus } \\
\text { (Schwein.) De Toni (1888) }\end{array}$ & FJ478125 & 0 & 0 & 2 & 0 & 0 & 2 \\
\hline & $\begin{array}{l}\text { Physalacriaceae } \\
\text { Flammulina mexicana }\end{array}$ & & & & & & & \\
\hline 44 & $\begin{array}{c}\text { Redhead, Estrada \& R.H. } \\
\text { Petersen }\end{array}$ & AF032129 & 1 & 0 & 0 & 0 & 0 & 1 \\
\hline 45 & $\begin{array}{l}\text { Oudemansiella canarii } \\
\text { (Jungh.) Höhn. (1909) }\end{array}$ & AY216473 & 1 & 0 & 2 & 1 & 0 & 4 \\
\hline 46 & $\begin{array}{c}\text { Pluteaceae } \\
\text { Pluteus romellii (Britzelm.) } \\
\text { Sacc } 1895 .\end{array}$ & HM562078 & 0 & 1 & 0 & 0 & 0 & 1 \\
\hline 47 & $\begin{array}{l}\text { Volvariella volvacea (Bul. } \\
\text { ex Fr.) Singer (1951) }\end{array}$ & HM246500 & 0 & 0 & 0 & 0 & 1 & 1 \\
\hline & Psathyrellaceae & & & & & & & \\
\hline & Coprinus fissolanatus & & & & & & & \\
\hline 48 & $\begin{array}{l}\text { Park,D.S., Shin,H.S. and } \\
\text { Moncalvo,J.M. } \\
\text { Coprinellus hiascens (Fr.) }\end{array}$ & AF345812 & 3 & 1 & 1 & 4 & 1 & 10 \\
\hline 49 & $\begin{array}{c}\text { Redhead, Vilgalys \& } \\
\text { Moncalvo } \\
\text { Coprinellus micaceus }\end{array}$ & JN159528 & 0 & 0 & 0 & 0 & 2 & 2 \\
\hline 50 & $\begin{array}{c}\text { (Bull.:Fr.) Vilgalys, Hopple } \\
\text { \& Jacq. Johnson } \\
\text { Coprinopsis lagopus }\end{array}$ & JN943116 & 1 & 1 & 0 & 1 & 0 & 3 \\
\hline 51 & $\begin{array}{c}\text { (Fries) Redhead, Vilgalys } \\
\text { \& Moncalvo }\end{array}$ & AF345815 & 2 & 0 & 1 & 1 & 0 & 3 \\
\hline 52 & $\begin{array}{l}\text { Coprinus sterquilinus (Fr.) } \\
\text { Fr. } 1838 . \\
\text { Parasola auricoma (Pat.) }\end{array}$ & FJ501551 & 0 & 1 & 1 & 0 & 0 & 2 \\
\hline 53 & $\begin{array}{c}\text { Redhead, Vilgalys \& } \\
\text { Hopple (2001). }\end{array}$ & JN943107 & 0 & 1 & 0 & 0 & 1 & 2 \\
\hline 54 & $\begin{array}{l}\text { Parasola conopilus (Fr.) } \\
\text { Örstadius \& E. Larss. }\end{array}$ & FJ770396 & 0 & 0 & 0 & 1 & 0 & 1 \\
\hline 55 & $\begin{array}{c}\text { Psathyrella bipellis (Quél.) } \\
\text { A.H.Sm. (1946) }\end{array}$ & FN430689 & 1 & 1 & 0 & 2 & 0 & 4 \\
\hline 56 & $\begin{array}{l}\text { Psathyrella candolleana } \\
\text { (Fr.) Maire (1937) }\end{array}$ & AB306311 & 0 & 0 & 1 & 0 & 0 & 1 \\
\hline
\end{tabular}


Ache et al., J. Appl. Biosci. 2019 Mushroom species richness, distribution and substrate specificity in the Kilum-ljim forest reserve of Cameroon

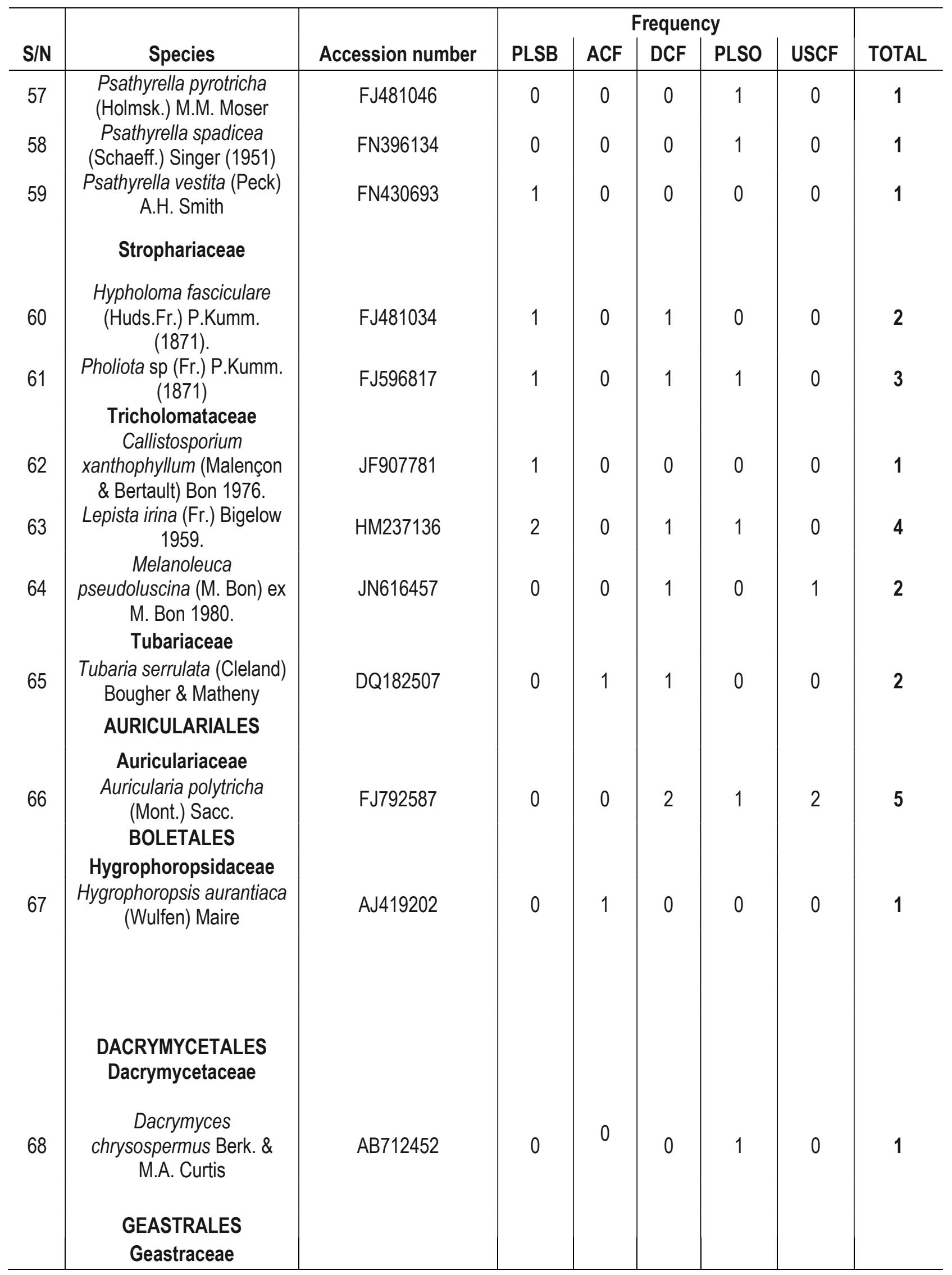


Ache et al., J. Appl. Biosci. 2019 Mushroom species richness, distribution and substrate specificity in the Kilum-ljim forest reserve of Cameroon

\begin{tabular}{|c|c|c|c|c|c|c|c|c|}
\hline \multirow[b]{2}{*}{$\mathrm{S} / \mathrm{N}$} & \multirow[b]{2}{*}{ Species } & \multirow[b]{2}{*}{ Accession number } & \multicolumn{5}{|c|}{ Frequency } & \multirow[b]{2}{*}{ TOTAL } \\
\hline & & & PLSB & ACF & DCF & PLSO & USCF & \\
\hline 69 & $\begin{array}{l}\text { Geastrum minimum } \\
\text { Schwein }\end{array}$ & EU784238 & 0 & 0 & 1 & 0 & 1 & 2 \\
\hline 70 & $\begin{array}{l}\text { Geastrum triplex Jungh. } \\
\text { GOMPHALES } \\
\text { Gomphaceae }\end{array}$ & JN942821 & 0 & 0 & 1 & 2 & 1 & 4 \\
\hline 71 & $\begin{array}{c}\text { Ramaria decurrens (Pers.) } \\
\text { R. H. Petersen }\end{array}$ & AJ408375 & 0 & 0 & 0 & 1 & 1 & 2 \\
\hline 72 & $\begin{array}{l}\text { Ramaria rubribrunnescens } \\
\text { Fr. ex Bonord. }\end{array}$ & EU652351 & 1 & 0 & 0 & 0 & 0 & 1 \\
\hline & $\begin{array}{l}\text { HELOTIALES } \\
\text { Helotiaceae } \\
\text { Chlorociboria }\end{array}$ & & & & & & & \\
\hline 73 & $\begin{array}{c}\text { aeruginascens (Nyl.) } \\
\text { Kanouse }\end{array}$ & JN943460 & 2 & 1 & 0 & 0 & 0 & 3 \\
\hline 74 & $\begin{array}{c}\text { Chlorociboria awakinoana } \\
\text { P.R.Johnst. }\end{array}$ & JN943462 & 1 & 0 & 0 & 0 & 0 & 1 \\
\hline & $\begin{array}{c}\text { Bionectriaceae } \\
\text { HYMENOCHAETALES } \\
\text { Hymenochaetaceae }\end{array}$ & & & & & & & \\
\hline 75 & $\begin{array}{c}\text { Phellinus repandus Quél. } \\
\text { Fuscoporia gilva }\end{array}$ & AF534076 & 0 & 1 & 0 & 1 & 0 & 2 \\
\hline 76 & $\begin{array}{c}\text { (Schwein.) T. Wagner \& M. } \\
\text { Fisch. }\end{array}$ & AM269795 & 0 & 0 & 0 & 1 & 0 & 1 \\
\hline & $\begin{array}{c}\text { HYPOCREALES } \\
\text { Bionectria ochroleuca }\end{array}$ & & & & & & & \\
\hline 77 & $\begin{array}{c}\text { (Schwein.) Schroers \& } \\
\text { Samuels } \\
\text { Cordycipitaceae }\end{array}$ & GU566253 & 1 & 0 & 0 & 0 & 0 & 1 \\
\hline 78 & $\begin{array}{l}\text { Cordyceps brongniartii } \\
\text { (Saccardo) Petch }\end{array}$ & AJ309349 & 0 & 0 & 1 & 0 & 0 & 1 \\
\hline 79 & $\begin{array}{c}\text { Cordyceps takaomontana } \\
\text { Fr. (1818) }\end{array}$ & AB189447 & 0 & 0 & 1 & 0 & 0 & 1 \\
\hline & $\begin{array}{l}\text { HYSTERANGIALES } \\
\text { Phallogastraceae } \\
\text { Protubera canescens }\end{array}$ & & & & & & & \\
\hline 80 & $\begin{array}{l}\text { G.W.Beaton \& Malajczuk } \\
(1986)\end{array}$ & GQ981520 & 2 & 0 & 0 & 0 & 0 & 2 \\
\hline & PEZIZALES & & & & & & & \\
\hline
\end{tabular}


Ache et al., J. Appl. Biosci. 2019 Mushroom species richness, distribution and substrate specificity in the Kilum-ljim forest reserve of Cameroon

\begin{tabular}{|c|c|c|c|c|c|c|c|c|}
\hline \multirow[b]{2}{*}{$S / N$} & \multirow[b]{2}{*}{ Species } & \multirow[b]{2}{*}{ Accession number } & \multicolumn{5}{|c|}{ Frequency } & \multirow[b]{2}{*}{ TOTAL } \\
\hline & & & PLSB & ACF & DCF & PLSO & USCF & \\
\hline 81 & $\begin{array}{c}\text { Pezizaceae } \\
\text { Peziza ostracoderma Dill. } \\
\text { ex Fries (1822) }\end{array}$ & JN002180 & 0 & 0 & 0 & 1 & 0 & 1 \\
\hline & $\begin{array}{l}\text { PHALLALES } \\
\text { Phallaceae }\end{array}$ & & & & & & & \\
\hline 82 & $\begin{array}{c}\text { Clathrus archeri (Berk.) } \\
\text { Dring 1980". }\end{array}$ & KP688386 & 0 & 0 & 0 & 1 & 0 & 1 \\
\hline 83 & $\begin{array}{c}\text { Clathrus ruber P.Micheli ex } \\
\text { Pers. (1801) }\end{array}$ & GQ981501 & 0 & 0 & 0 & 2 & 0 & 2 \\
\hline 84 & $\begin{array}{l}\text { Phallus impudicus } \\
\text { Linnaeus (1753) }\end{array}$ & AF324171 & 1 & 0 & 0 & 0 & 1 & 2 \\
\hline & $\begin{array}{l}\text { POLYPORALES } \\
\text { Fomitopsidaceae } \\
\text { Fomitopsis cajanderi }\end{array}$ & & & & & & & \\
\hline 85 & $\begin{array}{c}\text { (P.Karst.) Kotl. \& Pouzar } \\
\text { (1957) } \\
\text { Ganodermataceae }\end{array}$ & JQ673050 & 0 & 1 & 0 & 1 & 0 & 2 \\
\hline 86 & $\begin{array}{l}\text { Ganoderma applanatum } \\
\text { (Pers.) Pat. }\end{array}$ & AJ608709 & 2 & 1 & 0 & 0 & 0 & 3 \\
\hline 87 & $\begin{array}{c}\text { Ganoderma pfeifferi Bres. } \\
\text { Meripilaceae }\end{array}$ & AM906059 & 1 & 1 & 0 & 0 & 0 & 2 \\
\hline 88 & $\begin{array}{c}\text { Physisporinus vitreus } \\
\text { (Pers.) P.Karst. (1889) } \\
\text { Meruliaceae }\end{array}$ & JN182920 & 0 & 0 & 0 & 1 & 0 & 1 \\
\hline 89 & $\begin{array}{c}\text { Abortiporus biennis } \\
\text { (Schwein.) Murrill (1944) }\end{array}$ & FJ608589 & 0 & 0 & 0 & 1 & 0 & 1 \\
\hline 90 & Panus sp Fr. (1838) & HM245784 & 0 & 1 & 0 & 0 & 0 & 1 \\
\hline 91 & $\begin{array}{l}\text { Podoscypha petalodes } \\
\text { (Berk.) Boidin } \\
\text { Polyporaceae }\end{array}$ & AM773629 & 2 & 2 & 4 & 1 & 2 & 11 \\
\hline 92 & $\begin{array}{l}\text { Coriolopsis sanguinaria } \\
\text { (Klotzsch) Teng } 1963\end{array}$ & FJ627251 & 0 & 1 & 0 & 0 & 0 & 1 \\
\hline 93 & $\begin{array}{l}\text { Daedaleopsis confragosa } \\
\text { (Bolton) J.Schröt. (1888). }\end{array}$ & FJ810177 & 0 & 0 & 0 & 1 & 0 & 1 \\
\hline 94 & $\begin{array}{l}\text { Laetiporus sulphureus } \\
\text { (Bull.) Murrill (1920) }\end{array}$ & AY835667 & 0 & 0 & 2 & 0 & 1 & 3 \\
\hline 95 & $\begin{array}{l}\text { Lentinus squarrosulus } \\
\text { Mont. } 1842 .\end{array}$ & GU001951 & 1 & 2 & 1 & 2 & 0 & 6 \\
\hline 96 & $\begin{array}{c}\text { Lenzites elegans (Spreng.) } \\
\text { Pat. }\end{array}$ & HQ248217 & 0 & 1 & 0 & 0 & 0 & 1 \\
\hline 97 & $\begin{array}{l}\text { Microporus subaffinis } \\
\text { (Lloyd) Imazeki } 1943 .\end{array}$ & FJ627249 & 1 & 0 & 1 & 1 & 0 & 3 \\
\hline 98 & Polyporus arcularius & AB638344 & 1 & 0 & 1 & 1 & 0 & 3 \\
\hline
\end{tabular}


Ache et al., J. Appl. Biosci. 2019 Mushroom species richness, distribution and substrate specificity in the Kilum-ljim forest reserve of Cameroon

\begin{tabular}{|c|c|c|c|c|c|c|c|c|}
\hline \multirow[b]{2}{*}{ S/N } & \multirow[b]{2}{*}{ Species } & \multirow[b]{2}{*}{ Accession number } & \multicolumn{5}{|c|}{ Frequency } & \multirow[b]{2}{*}{ TOTAL } \\
\hline & & & PLSB & $\mathrm{ACF}$ & DCF & PLSO & USCF & \\
\hline 99 & $\begin{array}{c}\text { (Batsch) Fr. } \\
\text { Polyporus dictyopus Mont. } \\
1835 .\end{array}$ & AF516561 & 1 & 0 & 4 & 3 & 1 & 9 \\
\hline 100 & $\begin{array}{l}\text { Polyporus tenuiculus } \\
\text { (Beauv.) Fr. }\end{array}$ & JQ409357 & 2 & 2 & 1 & 1 & 1 & 7 \\
\hline 101 & $\begin{array}{c}\text { Skeletocutis nivea (Jungh.) } \\
\text { Keller. }\end{array}$ & JQ673120 & 0 & 0 & 1 & 0 & 0 & 1 \\
\hline 102 & $\begin{array}{c}\text { Trametes hirsuta (Wulfen) } \\
\text { Pilát }\end{array}$ & JN164952 & 1 & 0 & 1 & 1 & 0 & 3 \\
\hline 103 & $\begin{array}{c}\text { Trametes polyzona (Pers.) } \\
\text { Corner }\end{array}$ & JN164980 & 0 & 0 & 1 & 0 & 0 & 1 \\
\hline 104 & $\begin{array}{l}\text { Trametes sanguinea (L.) } \\
\text { Imazeki }\end{array}$ & JN164981 & 1 & 0 & 1 & 1 & 1 & 4 \\
\hline 105 & $\begin{array}{c}\text { Trametes versicolor (L.) } \\
\text { Lloyd (1920) }\end{array}$ & EU153514 & 1 & 1 & 2 & 1 & 0 & 5 \\
\hline & RUSSULALES & & & & & & & \\
\hline 106 & $\begin{array}{c}\text { Lachnocladiaceae } \\
\text { Lachnocladium sp Lév. } \\
(1846)\end{array}$ & DQ192176 & 0 & 0 & 0 & 0 & 1 & 1 \\
\hline 107 & $\begin{array}{c}\text { Stereaceae } \\
\text { Stereum hirsutum (Willd.) } \\
\text { Pers. (1800). } \\
\text { Stereum sanquinolentum }\end{array}$ & AM269810 & 1 & 0 & 1 & 0 & 2 & 4 \\
\hline 108 & $\begin{array}{c}\text { (Alb. \& Schwein.) Fr. } \\
\text { (1838). } \\
\text { XYLARIALES }\end{array}$ & EU673084 & 0 & 0 & 1 & 0 & 1 & 2 \\
\hline & $\begin{array}{c}\text { Xylariaceae } \\
\text { Daldinia concentrica }\end{array}$ & & & & & & & \\
\hline 109 & $\begin{array}{l}\text { (Bolton) Cesati \& de } \\
\text { Notaris } \\
\text { Podosordaria muli J.D. }\end{array}$ & AF163021 & 1 & 0 & 1 & 0 & 0 & 2 \\
\hline 110 & $\begin{array}{c}\text { Rogers, Y.M. Ju \& F. San } \\
\text { Martín }\end{array}$ & GU324761 & 0 & 0 & 0 & 1 & 0 & 1 \\
\hline 111 & $\begin{array}{c}\text { Xylaria sp MUCL } 51605 \\
\text { Hill ex Schrank (1789) }\end{array}$ & FN689802 & 1 & 1 & 2 & 2 & 2 & 8 \\
\hline 112 & $\begin{array}{c}\text { Xylaria adscendens (Fr.) } \\
\text { Fr., } 1851 .\end{array}$ & GU322432 & 1 & 0 & 2 & 1 & 2 & 6 \\
\hline 113 & $\begin{array}{c}\text { Xylaria bambusicola Y.M. } \\
\text { Ju \& J.D. Rogers }\end{array}$ & GU300088 & 0 & 0 & 0 & 1 & 1 & 2 \\
\hline 114 & Xylaria curta Fries & GU322444 & 1 & 0 & 1 & 2 & 1 & 5 \\
\hline 115 & $\begin{array}{l}\text { Xylaria grammica (Mont.) } \\
\text { Mont. }\end{array}$ & AB524025 & 0 & 0 & 1 & 1 & 0 & 2 \\
\hline \multirow[t]{2}{*}{116} & $\begin{array}{l}\text { Xylaria ianthinovelutina } \\
\text { (Mont.) Mont. }\end{array}$ & GU322441 & 0 & 0 & 0 & 1 & 0 & 1 \\
\hline & TOTAL & & 60 & 35 & 77 & 86 & 45 & 303 \\
\hline
\end{tabular}




\section{Ache et al., J. Appl. Biosci. 2019 Mushroom species richness, distribution and substrate specificity in the Kilum-ljim forest reserve of Cameroon}

The diversity and similarity indices between sampling sites are presented on Table 6 and 7 respectively. High diversity was observed in all the study sites, though a weak similarity was observed in all the study sites. Plantlife sanctuary Oku and Plantlife sanctuary Belo were more similar than all the other sites.

Table 6: Site variation in the species diversity of macrofungi in the Kilum-ljim forest

\begin{tabular}{c|c}
\hline Sampling site & Simpson Diversity Index \\
\hline PLSB & 0.96 \\
\hline ACF & 0.94 \\
\hline DCF & 0.96 \\
\hline PLSO & 0.96 \\
\hline USCF & 0.94 \\
\hline
\end{tabular}

PLSB= Plantlife sanctuary Belo, ACF= Anyajua community forest, $\mathrm{DCF}=$ Dichami community forest, $\mathrm{PLSO}=$ Plantlife sanctuary Oku, USCF= Upper-shinga community forest

Table 7: Jaccard's similarity matrix among different sampling sites within Kilum-ljim forest

\begin{tabular}{c|c|c|c|c|c}
\hline & PLSB & ACF & DCF & PLSO & USCF \\
\hline PLSB & 1 & 0.257 & 0.391 & 0.408 & 0.224 \\
\hline ACF & & 1 & 0.188 & 0.211 & 0.138 \\
\hline DCF & & & 1 & 0.298 & 0.277 \\
\hline PLSO & & & & 1 & 0.167 \\
\hline USCF & & & & & 1
\end{tabular}

PLSB= Plantlife sanctuary Belo, ACF= Anyajua community forest, DCF= Dichami community forest, $P L S O=$ Plantlife sanctuary Oku, USCF= Upper-shinga community forest

Substratum of molecularly identified macrofungi: The substrata of the molecularly identified macrofungi in this study by Teke et al. (2017) in the Kilum-ljim forest were recorded (Table 8). Majority of the macrofungi occurred on dead wood and accounts for high decomposition rate in forest wood while few macrofungi occurred on dung.

Table 8: Occurrence of macrofungi on different substrata in the Kilum-ljim forest

\begin{tabular}{l|l|l|l}
\hline S/N & Species & Family & Substratum \\
\hline & AGARICALES & & \\
1 & Agaricus litoralis (Wakef. \& A. Pearson) Pilat. & Agaricaceae & Dung \\
2 & Agaricus xanthodermus Genev. (1876) & Agaricaceae & Dung \\
3 & Cystolepiota hetieri (Boud.) Singer & Agaricaceae & Leaf litter \\
4 & Lepiota sp PA620 (Pers.) Gray (1821) & Agaricaceae & Leaf litter \\
5 & Leucoagaricus cupresseus (Burl.) Boisselet \& Guinb. & Agaricaceae & Soil \\
6 & Leucoagaricus flavovirens J.F. Liang, Zhu L. Yang \& J. Xu & Agaricaceae & Soil \\
7 & Leucoagaricus gaillardia Bon \& Boiffard 1974 & Agaricaceae & Soil \\
8 & Leucoagaricus littoralis(Menier) Bon \& Boiffard 1970 & Agaricaceae & Soil \\
9 & Leucoagaricus rubrotinctus(Peck) Singer (1948) & Agaricaceae & Soil \\
10 & Leucoagaricus serenus(Fr.) Bon \& Boiffard 1974 & Agaricaceae & Soil \\
11 & Leucoagaricus viriditinctus (Berk. \& Broome) J.F. & Agaricaceae & Soil \\
12 & Macrolepiota dolichaula (Berk. \& Broome) Pegler \& R.W. Rayner & Agaricaceae & Soil \\
13 & Vascellum pretense(Pers.) Kreise & Agaricaceae & Leaf litter \\
14 & Panaeolus foenisecii (Pers.) R.Maire (1933). & Bolbitiaceae & Leaf litter \\
15 & Panaeolus sphinctrinus (Fr.) Quél. & Bolbitiaceae & Leaf litter \\
16 & Crepidotus epibryus (Fr.) Quél. 1888 & Crepidotaceae & Dead wood \\
\hline
\end{tabular}


Ache et al., J. Appl. Biosci. 2019 Mushroom species richness, distribution and substrate specificity in the Kilum-ljim forest reserve of Cameroon

\begin{tabular}{|c|c|c|c|}
\hline $\mathbf{S} / \mathbf{N}$ & Species & Family & Substratum \\
\hline 17 & Crepidotus mollis (Schaeff.) Staude 1857 & Crepidotaceae & Dead wood \\
\hline 18 & Entoloma araneosum (Quél.) M.M. Moser. & Entolomataceae & Leaf litter \\
\hline 19 & Camarophyllus pratensis (Pers.) P. Kumm. & Hygrophoraceae & Soil \\
\hline 20 & Hygrocybe helobia (Arnolds) Bon & Hygrophoraceae & Soil \\
\hline 21 & Hygrocybe persistens (Britzelmayr) Singer & Hygrophoraceae & Soil \\
\hline 22 & Galerina badipes (Pers.) Kühner & Hymenogastraceae & Dead wood \\
\hline 23 & Galerina hybrid Kühner. & Hymenogastraceae & Dead wood \\
\hline 24 & Galerina marginata (Batsch) Kühner (1935) & Hymenogastraceae & Dead wood \\
\hline 25 & Psilocybe cubensis (Earle) Singer & Hymenogastraceae & Soil \\
\hline 26 & Lyophyllum connatum P. Karst. & Lyophyllaceae & Soil \\
\hline 27 & Termitomyces microcarpus (Berk.\&Broome) R. Heim & Lyophyllaceae & Soil \\
\hline 28 & Termitomyces striatus (Beeli) R. Heim & Lyophyllaceae & Soil \\
\hline 29 & Termitomyces sp V1PR. Heim & Lyophyllaceae & Soil \\
\hline 30 & Termitomyces sp Group8R.Heim & Lyophyllaceae & Soil \\
\hline 31 & Clitocybula lacerate (Scop.) Singer ex Métrod & Marasmiaceae & Dead wood \\
\hline 32 & Clitocybula oculus(Peck) Singer 1962 & Marasmiaceae & Dead wood \\
\hline 33 & Crinipellis scabella (Alb. \& Schwein.) Murrill & Marasmiaceae & Dead wood \\
\hline 34 & Hydropus marginellus (Pers. : Fr.) Singer 1948 & Marasmiaceae & Dead wood \\
\hline 35 & Marasmius purpureostriatus Hongo 1958 & Marasmiaceae & Dead wood \\
\hline 36 & Marasmiellus ramealis (Bull.) Singer & Marasmiaceae & Dead wood \\
\hline 37 & Marasmius rotula (Scop.) Fr. & Marasmiaceae & Dead wood \\
\hline 38 & Favolaschia calocera R. Heim & Mycenaceae & Dead wood \\
\hline 39 & Mycena acicula (Schaeff.) P.Kumm. (1871) & Mycenaceae & Dead wood \\
\hline 40 & Mycena laevigata(Lasch) Gillet & Mycenaceae & Dead wood \\
\hline 41 & Mycena pura (Pers.) P. Kumm. & Mycenaceae & Dead wood \\
\hline 42 & Panellus stipticus (Bull.) P.Karst. (1879) & Mycenaceae & Dead wood \\
\hline 43 & Cyathus stercoreus (Schwein.) De Toni (1888) & Nidulariaceae & Dead wood \\
\hline 44 & Flammulina mexicana Redhead, Estrada \& R.H. Petersen & Physalacriaceae & Dead wood \\
\hline 45 & Oudemansiella canarii (Jungh.) Höhn. (1909) & Physalacriaceae & Dead wood \\
\hline 46 & Pluteus romellii (Britzelm.) Sacc 1895. & Pluteaceae & Leaf litter \\
\hline 47 & Volvariella volvacea (Bul. ex Fr.) Singer (1951) & Pluteaceae & Leaf litter \\
\hline 48 & Coprinus fissolanatus Park,D.S., Shin,H.S. and Moncalvo,J.M. & Psathyrellaceae & Leaf litter \\
\hline 49 & Coprinellus hiascens(Fr.) Redhead, Vilgalys \& Moncalvo & Psathyrellaceae & Leaf litter \\
\hline 50 & Coprinellus micaceus (Bull.:Fr.) Vilgalys, Hopple \& Jacq. Johnson & Psathyrellaceae & Leaf litter \\
\hline 51 & Coprinopsis lagopus (Fries) Redhead, Vilgalys \& Moncalvo & Psathyrellaceae & Leaf litter \\
\hline 52 & Coprinus sterquilinus (Fr.) Fr. 1838. & Psathyrellaceae & Leaf litter \\
\hline 53 & Parasola auricoma (Pat.) Redhead, Vilgalys \& Hopple (2001). & Psathyrellaceae & Leaf litter \\
\hline 54 & Parasola conopilus (Fr.) Örstadius \& E. Larss. & Psathyrellaceae & Leaf litter \\
\hline 55 & Psathyrella bipellis (Quél.) A.H.Sm. (1946) & Psathyrellaceae & Leaf litter \\
\hline 56 & Psathyrella candolleana (Fr.) Maire (1937) & Psathyrellaceae & Leaf litter \\
\hline 57 & Psathyrella pyrotricha (Holmsk.) M.M. Moser & Psathyrellaceae & Leaf litter \\
\hline 58 & Psathyrella spadicea (Schaeff.) Singer (1951) & Psathyrellaceae & Leaf litter \\
\hline 59 & Psathyrella vestita(Peck) A.H. Smith & Psathyrellaceae & Leaf litter \\
\hline 60 & Hypholoma fasciculare (Huds.Fr.) P.Kumm. (1871). & Strophariaceae & Leaf litter \\
\hline 61 & Pholiota sp (Fr.) P.Kumm. (1871) & Strophariaceae & Dung \\
\hline
\end{tabular}


Ache et al., J. Appl. Biosci. 2019 Mushroom species richness, distribution and substrate specificity in the Kilum-ljim forest reserve of Cameroon

\begin{tabular}{|c|c|c|c|}
\hline $\mathrm{S} / \mathrm{N}$ & Species & Family & Substratum \\
\hline 62 & Callistosporium xanthophyllum (Malençon \&Bertault) Bon 1976. & Tricholomataceae & Soil \\
\hline 63 & Lepista irina (Fr.) Bigelow 1959. & Tricholomataceae & Soil \\
\hline 64 & Melanoleuca pseudoluscina (M. Bon) ex M. Bon 1980. & Tricholomataceae & Soil \\
\hline 65 & Tubaria serrulata(Cleland) Bougher \& Matheny & Tubariaceae & Soil \\
\hline 66 & Auricularia polytricha (Mont.) Sacc. & Auriculariaceae & Deadwood \\
\hline 67 & Hygrophoropsis aurantiaca(Wulfen) Maire & Hygrophoropsidaceae & Dead wood \\
\hline 68 & Dacrymyces chrysospermus Berk. \& M.A. Curtis & Dacrymycetaceae & Dead wood \\
\hline 69 & Geastrum minimum Schwein & Geastraceae & Leaf litter \\
\hline 70 & Geastrum triplex Jungh. & Geastraceae & Leaf litter \\
\hline 72 & Ramaria rubribrunnescens Fr. ex Bonord. & Gomphaceae & Soil \\
\hline 73 & Chlorociboria aeruginascens (Nyl.) Kanouse & Helotiaceae & Deadwood \\
\hline 74 & Chlorociboria awakinoana P.R.Johnst. & Helotiaceae & Deadwood \\
\hline 75 & Phellinus repandus Quél. & Hymenochaetaceae & Dead wood \\
\hline 76 & Fuscoporia gilva (Schwein.) T. Wagner \& M. Fisch. & Hymenochaetaceae & Dead wood \\
\hline 77 & Bionectria ochroleuca (Schwein.) Schroers \& Samuels & Bionectriaceae & Soil \\
\hline 78 & Cordyceps brongniartii (Saccardo) Petch & Cordycipitaceae & Soil \\
\hline 79 & Cordyceps takaomontana Fr. (1818) & Cordycipitaceae & Soil \\
\hline 81 & Peziza ostracoderma Dill. ex Fries (1822) & Pezizaceae & Soil \\
\hline 82 & Clathrus archeri (Berk.) Dring 1980". & Phallaceae & Leaf litter \\
\hline 83 & Clathrus ruber P.Micheli ex Pers. (1801) & Phallaceae & Leaf litter \\
\hline 84 & Phallus impudicus Linnaeus (1753) & Phallaceae & Leaf litter \\
\hline 85 & Fomitopsis cajanderi (P.Karst.) Kotl. Pouzar (1957) & Fomitopsidaceae & Leaf litter \\
\hline 86 & Ganoderma applanatum (Pers.) Pat. & Ganodermataceae & Dead wood \\
\hline 87 & Ganoderma pfeifferi Bres. & Ganodermataceae & Dead wood \\
\hline 88 & Physisporinus vitreus (Pers.) P.Karst. (1889) & Meripilaceae & Dead wood \\
\hline 89 & Abortiporus biennis (Schwein.) Murrill (1944) & Meripilaceae & Dead wood \\
\hline 90 & Panus sp Fr. (1838) & Meripilaceae & Dead wood \\
\hline 91 & Podoscypha petalodes (Berk.) Boidin & Meripilaceae & Dead wood \\
\hline 92 & Coriolopsis sanguinaria(Klotzsch) Teng 1963 & Polyporaceae & Dead wood \\
\hline 93 & Daedaleopsis confragosa (Bolton) J.Schröt. (1888). & Polyporaceae & Dead wood \\
\hline 94 & Laetiporus sulphureus (Bull.) Murrill (1920) & Polyporaceae & Dead wood \\
\hline 95 & Lentinus squarrosulus Mont. 1842. & Polyporaceae & Dead wood \\
\hline 96 & Lenzites elegans (Spreng.) Pat. & Polyporaceae & Dead wood \\
\hline 97 & Microporus subaffinis (Lloyd) Imazeki 1943. & Polyporaceae & Dead wood \\
\hline 98 & Polyporus arcularius(Batsch) Fr. & Polyporaceae & Dead wood \\
\hline 99 & Polyporus dictyopus Mont. 1835. & Polyporaceae & Dead wood \\
\hline 100 & Polyporus tenuiculus (Beauv.) Fr. & Polyporaceae & Dead wood \\
\hline 101 & Skeletocutis nivea (Jungh.) Keller. & Polyporaceae & Dead wood \\
\hline 102 & Trametes hirsute (Wulfen) Pilát & Polyporaceae & Dead wood \\
\hline 103 & Trametes polyzona (Pers.) Corner & Polyporaceae & Dead wood \\
\hline 104 & Trametes sanguinea (L.) Imazeki & Polyporaceae & Dead wood \\
\hline 105 & Trametes versicolor (L.) Lloyd (1920) & Polyporaceae & Dead wood \\
\hline 106 & Lachnocladium sp Lév. (1846) & Lachnocladiaceae & Dead wood \\
\hline 107 & Stereum hirsutum (Willd.) Pers. (1800). & Stereaceae & Dead wood \\
\hline 108 & Stereum sanguinolentum (Alb. \& Schwein.) Fr. (1838). & Stereaceae & Dead wood \\
\hline
\end{tabular}




\begin{tabular}{|c|c|c|c|}
\hline \multicolumn{4}{|c|}{$\begin{array}{l}\text { Ache et al., J. Appl. Biosci. } 2019 \text { Mushroom species richness, distribution and substrate specificity in the } \\
\text { Kilum-ljim forest reserve of Cameroon }\end{array}$} \\
\hline $\mathrm{S} / \mathrm{N}$ & Species & Family & Substratum \\
\hline 109 & Daldinia concentric (Bolton) Cesati \& de Notaris & Xylariaceae & Standingwood \\
\hline 110 & Podosordaria muli J.D. Rogers, Y.M. Ju \& F. San Martín & Xylariaceae & Dead wood \\
\hline 111 & Xylaria sp MUCL 51605 Hill ex Schrank (1789) & Xylariaceae & Dead wood \\
\hline 112 & Xylaria adscendens (Fr.) Fr., 1851. & Xylariaceae & Dead wood \\
\hline 113 & Xylaria bambusicola Y.M. Ju \& J.D. Rogers & Xylariaceae & Dead wood \\
\hline 114 & Xylaria curta Fries & Xylariaceae & Dead wood \\
\hline 115 & Xylaria grammica(Mont.) Mont. & Xylariaceae & Dead wood \\
\hline 116 & Xylaria ianthinovelutina(Mont.) Mont. & Xylariaceae & Dead wood \\
\hline
\end{tabular}

\section{DISCUSSION}

Macrofungi diversity was high in all the five forest sites sampled. The Plantlife Sanctuaries in Belo and Oku recorded the highest species diversity as well as species richness. This was probably due to the fact that these forests are restricted sites to the population and there is little disturbance. The community forests (Anyajua community forest and Upper-shinga community forest) with the exception of Dichami community forest, recorded lower diversity and were very low in species richness. This could be explained by the fact that these sites are more exposed and accessible to the community members and as such liable to large disturbances. These results are similar to the findings of Brown et al. (2006) and Douanla-Meli, (2007) who reported that habitat degradation and forest fragmentation were threats to macrofungal diversity in Western Ghats of India and in the Mbalmayo forest reserve in Cameroon respectively. The high macrofungi diversity in Dichami community forest could be due to the fact the inhabitants around this forest are the Fulanis who mostly carry out mostly crazing instead of farming and other anthropogenic activities. A relative high species similarity index was observed between Plantlife Sanctuary in Belo and Plantlife Sanctuary in Oku. This could probably be due to the fact that these sites are restricted to community members. Results also revealed that out of the 116 species identified molecularly, $12 \%$ (14) occurred in all or four sites while $47 \%$ (54) species occurred only in one site. This result could be explained by the fact that the macrofungal diversity is gradually reducing and some of these species risk being extinct even before they are identified. The highest occurring and most frequent species belong to the genera Favolaschia, Leucoagaricus, Marasmius, Polyporus, Lentinus, Podoscypha and Termitomyces. Some genera like Coprinus, Lepiota, Crepidotus, Ganoderma, Geastrum, Ramaria, Marasmius, Mycena, Polyporus, Trametes and Xylaria have been reported in previous works in
Cameroon by Kinge et al. (2013) and Douanla-Meli (2007). The order Agaricales was the most represented taxa $(56 \%)$ recorded during this study. This is broadly consistent with previous works of Isikhuemhen, (2000) in his study on the first International mushroom foray in Nigeria, and Egbe et al. (2013) in their study on the diversity and distribution of macrofungi in the mount Cameroon region, and Yanxin et al. (2016) in their study on macrofungal diversity in Yaoluoping Nature Reserve Anhui China. Members of the polyporaceae represented the highest number of species. This is in line with different studies carried out by Tadiosa et al. (2011), De Leon et al. (2013) and Rajput et al. (2015), who all reported higher numbers of species belonging to Polyporaceae in the province of Aurora, Central Luzon, Philippines and Gujarat State India, respectively. These findings are probably justified by the fact that most wood inhabiting species are polypores. Seasonal variations recorded vast changes in macrofungi fruiting. The month of September however recorded the highest macrofungal fructification. These results are broadly consistent with those of Lagana et al. (2002), Salerni et al. (2002), Sibounnavong et al. (2008), Mani and Kumaresan (2009) and Baptista et al. (2010) who all reported that rainfall favors diversity and productivity of macrofungi fruit bodies. From the substratum point of view, majority of the macrofungi were recorded from dead wood $(56 \%)$ followed by leaf litter $(26 \%)$, soil $(17 \%)$, cow dung $(1 \%)$ and standing stem (1\%). This high occurrence on dead wood is probably due to the fact that the habitat is forest which favors wood inhabiting macrofungi. This probably accounts for the high decomposition rate in forest wood. These results are similar with the findings of Brown et al. (2006) who reported that species occurrence in different substrata may probably be due to the environmental conditions and habitat. To the best of our knowledge, this is the first study to examine macrofungi species richness, 
distribution and substrate specificity in the Kilum-ljim forest. It is worth noting that the genera Abortiporus, Callistosporium, Coprinellus, Coprinopsis, Cordyceps, Cystolepiota, Chlorociboria, Crinipellis, Clathrus,

\section{CONCLUSION}

Plantlife Santuary Oku had the highest number of mushroom species and the least was the Anyajua community forest. Podoscypha petalodes had the highest number of species across all the five sites surveyed followed by Coprinus fissolanatus, Polyporus dictopus, Favolachia calocera and Xylaria sp. The highest collection of macrofungi was recorded in September while the least was in January. The substrate specificity general trend showed that fungi prefer certain specific substrates. Deadwood and leaf litter substrate supported more macrofungi while dung and standing stem substrates supported the least

\section{ACKNOWLEDGEMENT}

The authors gratefully acknowledge funding from the Rufford Small Grant for nature conservation. All the

\section{REFERENCES}

Asanga CA, 2001. Facilitating Viable Partnership in Community Forest Management in Cameroon: The Case of the Kilum-ljim Montain Forest Area. In: Wallenberg, E., Edmunds, D., Buck, L., Fox, J., Brodt, S. (2001). Social Learning in Community Forests. CIFOR, Indonesia, pp. 1209.

Amaranthus MP, 1998. The importance and conservation of ectomycorrhizal fungal diversity in forest ecosystems: lessons from Europe and the Pacific Northwest. Portland, OR, USA, USDA For. Serv., Gen.Tech. Rep. PNW-GTR-431: $15 \mathrm{p}$.

Baptista, P., Martins, A., Taveres, R. M. and Lino-Neto, $T, 2010$. Diversity and fruiting pattern of Macrofungi associated with chestnut (Castanea sativa) in the Tras-os-Montes region (Northeast Portugal). Fungal Ecology 3: 9-19.

Boa ER, 2004. Wild Edible Fungi: A Global Overview of Their Use and Importance to People Non Wood Forest Products 17. FAO Publishing Management Services, Rome 157pp.

Brown N, Bhagwat S, Watkinson S, 2006. Macrofungal diversity in fragmented and disturbed forests of the Western Ghats of India. Journal of Applied Ecology 43: 11-17.
Galerina, Laetiporus, Melanoleuca, Panaeolus, Parasola, Podosordaria, Physisporinus, Skeletocutis and Tubaria are new records to the Cameroon macrofungi literature.

macrofungi. The Agaricaceae and Polyporaceae were more abundant while Entolomataceae, Tuberiaceae, Auriculariaceae, Hygrophoropsidaceae, Dacrymy cetaceae, Gomphaceae, Bioectriaceae, Pezizaceae, Fomitopsidaceae and Lechnocladiaceae were represented by a single species only. This study adds to the baseline data of macrofungi diversity, distribution and substrate specificity in Cameroon. The data especially on the wood rotting fungi will be an indicator of ecological continuity of forest at the studied area and will be very useful for recording future changes in the climate, vegetation and air composition.

community leaders and field guides are appreciated for assistance.

De Leon AM, Luangsa-ard JJD, Karunarathna SC, Hyde KD, Reyes RG, Dela-Cruz TEE, 2013. Species listing, distribution, and molecular identification of macrofungi in six Aeta tribal communities in Central Luzon, Philippines. Mycosphere 4(3):478-494.

Douanla-Meli C, 2007. Fungi of Cameroon: ecological diversity with emphasis on the taxonomy of non-gilled Hymenomycetes from the Mbalmayo Forest Reserves. Bibliotheca Mycologica, 410.

Egbe EA, Tonjock RK, Ebai MT, Nji T, Mih A M, 2013. Diversity and distribution of Macrofungi (Mushrooms) in the Mount Cameroon Region. Journal of Ecology and Natural Environment 5 (10):310-334.

Eneke ETB, 2011. Growth and in vitro phosphate solubilizing ability of Scleroderma sinnamariense: A tropical mycorrhizal fungus isolated from Gnetum africanum ectomycorrhiza root tips. Journal of Yeast and Fungal Research 2 (9): 132-142.

Fomété T, Vermaat, J, Gardner A, DeMarco J, Asanga C, Tekwe C, Percy F, 2001. A Conservation Partnership: Community Forestry at Kilum-Jjim, Cameroon, Rural Development Forestry 
Network, Russell Press Ltd, Nottingham, UK, ISBN 085003 5392, pp. 9-16.

Forboseh P. and Maisels F, 2000. The Kilum-ljim forest survey: Ecological Monitoring Programme KIFP, BirdLife International/MINEF, Cameroon.

Gates GM, 2009. Coarse woody debris, macrofungal assemblages, and sustainable forest management in a Eucalyptus oblique forest of southern Tasmania. Ph.D. thesis, University of Tasmania, Hobart, Tasmania, Australia 370pp.

Gardner AA, De-Marco J, Asanga, CA, 2001. A conservation partnership: Community Forestry at Kilum-ljim, Cameroon. In: Rural Forestry Network. 17pp.

Hawksworth DL, 1991. The fungal dimension of biodiversity: magnitude, significance, and conservation. Mycological Research 95: 641655.

Isikhuemhen OS, 2000. First International mushroom foray in Nigeria. Inoculum 51: 9-10

Kinge TR, Mih AM, Coetzee MPA, 2012. Molecular phylogenetic relationships among species of Ganoderma in Cameroon. Australian Journal of Botany 60: 526-538

Kinge TR, Egbe EA, Tabi EM, Nji TM, Mih AM, 2013. The first checklist of macro fungi of Mount Cameroon. Mycosphere 4: 694-699.

Krebs CJ, 2014. Ecological methodology (3rd edition). Benjamin Cummings, University of British Columbia, Vancouver. 768pp.

Lagana A, Angiolini C, Loppi S, Salerni E, Perini C, Barluzzi C, De Dominicis V, 2002. Periodicity, fluctuations and successions of macrofungi in fir forest (Abies Alba Miller) in Tuscany, Italy. Forest Ecology and Management 169: 187202.

Lindequist $U$, Niedermeyer T, Jülich W, 2005. The pharmacological potential of mushrooms. Evidence-Based Complementary and Alternative Medicine 2 (3): 285-299.

Lloyd M and Ghelardi RJ, 1964. A table calculating the "equitability" component of species diversity. Journal of Animal Ecology 33 (2): 217-225.

Lodge JD, Ammirati FJ, O'Dell ET, Mueller MJ, 2004. Collecting and Describing Macrofungi. Pages 128-158, In: Biodiversity of Fungi: Inventory and Monitoring Methods, Mueller, M.G., F.G. Bills and S.M. Foster (Eds.). Elsevier Academic Press, San Diego, CA.
Mani S. and Kumaresan V, 2009. Occurrence of macrofungi on the coromandel coast of Tamil Nadu, Southern India. Journal of Threatened Taxa 641 (1): 54-57.

Mclntosh RP, 1967. An index of diversity and the relation of certain concepts to diversity. Ecology 48: 392-404.

Molina R, Pilz D, Smith J, Dunham S, Dreisbach T, O'Dell T, Castellano M, 2008. Conservation and management of forest fungi in the Pacic Northwestern United States: an integrated ecosystem approach. In: Moore D, Nauta NN, Evans SE, Rotheroe $M$, editors. Fungal conservation: issues and solutions. Cambridge: Cambridge University, pp. 19-63.

Mossebo DC, Njouonkou AL, Piatek M, Ayissi KB, Djamndo DM, 2009. Termitomyces striatus $f$. pileatus $f$. Nov. and $f$. brunneus f. nov. from Cameroon with a key to central African species. Mycotaxon 107: 415-329.

Mossobo DC, Essouman EPF, Machourt MC, Gueidan C, 2017. Phylogenetic relationships, taxonomic revision and new taxa of Termitomyces (Lyophyllaceae, Basidiomycota) inferred from combined nLSU- and mtSSUrDNA sequences. Phytotaxa 321, DOI: http://dx.doi.org/10.11646/phytotaxa.321.1.3

Mueller GM, Bills GF, Foster MS, 2004. Biodiversity of Fungi: Inventory and monitoring methods. Elsevier Academic press, Burlington, MA. 777pp.

Mueller GM, Schmit JP, Leacock PR, Buyck B, Cifuentes DJ, Kurt H, Teresa I, Karl-Henrik LD, Jean L, Tom WM, David M, Mario R, Scott AR, Leif R., James, MT, Roy W, Qiuxin W, 2007. Global diversity and distribution of macrofungi. Biodiversity and Conservation 16: 37-48.

Onguene NA, Kuyper TW, 2001. Mycorrhizal association in the rain forest of south Cameroon. Forest Ecology and Management 140: 277-287.

Osemwegie OO, Eriyaremu EG, Abdulmalik J, 2006. A survey of macrofungi in Edo/Delta region of Nigeria, their morphology and uses. Global Journal of Pure Applied Sciences 12: 149-157.

Pacioni G. and Lincoff GH, 1981. Simon and Schusters guide to mushrooms. Simon and Schusters Inc. New York, U.S.A. 154pp.

Rajput KS, Koyani RD, Patel, HP, Vasava AM, Patel RS, Patel AD, Singh AP, 2015. Preliminary checklist of fungi of Gujarat State, India. 
Current Research in Environmental and Applied Mycology 5(4):285-306.

Robert P. and Ryvarden L, 2006. Poroid fungi from the Korup National Park, Cameroon. Kew Bulletin 61:55-78.

Salerni E, Lagana A, Perini C, Loppi S, De Dominicis V, 2002. Effects of temperature and rainfall on fruiting of macrofungi in oak forest of the Mediterranean area. Israel Journal of Plant Sciences 50: 189-198.

Seen-Irlet B, Heilmann-Clausen J, Genney D, Dahlberg A, 2007. Guidance for the conservation of mushrooms in Europe. Convention on the conservation of European wildlife and natural habitats $27^{\text {th }}$ meeting, Strasbourg pp. 1-34.

Sibounnavong P, Cynthia CD, Kalaw SP, Reyes RG, Saytong K, 2008. Some species of Macrofungi at Puncan, Carranglan, Nueva Ecija in the Phillipines. Journal of Agricultural Technology 4(2):105-115.

Tadiosa ER, Agbayani ES, Agustin NT, 2011. Preliminary Study on the Macrofungi of Bazal Baubo Watershed, Aurora Province, Central Luzon, Philippines. Asian Journal of Biodiversity 2:149-171

Teke NA, Kinge TR, Bechem E, Mih AM, Kyalo M, Stomeo F, 2017. Macro-fungal diversity in the Kilum-ljim forest, Cameroon. Studies in Fungi 2(1): 47-58

Tibuhwa DD, 2011. Substrate specificity and phenology of macrofungi community at the University of Dar es Salaam main campus, Tanzania. Journal of Applied Biosciences 46: 3173-3184.

Kinge TR, Nkengmo AA, Nji TM, Ache NA, Mih AM, 2017. Species Richness and Traditional Knowledge of Macrofungi (Mushrooms) in the Awing Forest Reserve and Communities, Northwest Region, Cameroon. Journal of Mycology, https://doi.org/10.1155/2017/2809239

Yanxin HE, Wangboa WU, Nengshu LI, 2016. A checklist of Macrofungi in Yaoluoping Nature Reserve, Anhui. Journal of Resources and Ecology 7(2):144-150. 